\title{
OS NANOMATERIAIS E A DESCOBERTA DE NOVOS MUNDOS NA BANCADA DO QUÍMICO
}

\author{
Manuel A. Martins e Tito Trindade* \\ Departamento de Química, Universidade de Aveiro, 3810-193 Aveiro, Portugal
}

Recebido em 3/10/11; aceito em 17/2/12; publicado na web em 15/6/12

\begin{abstract}
NANOMATERIALS AND THE DISCOVERY OF NEW WORLDS AT THE CHEMIST'S BENCH. In recent years there has been great progress in the field of nanotechnology largely driven by research into nanomaterials. Chemistry appears in this context for its relevant role in the synthesis and surface modification of nanomaterials. This review article discusses fundamental concepts related to the synthesis and properties of inorganic nanoparticles with diverse properties. Aspects related to unique size dependent optical and magnetic properties are discussed and the chemistry involved in the preparation of nanomaterials reviewed. Fundamental aspects of the chemical modification of nanoparticles envisaging potential applications for these materials are also addressed.
\end{abstract}

Keywords: nanoparticles; chemical synthesis; surface modification.

\section{INTRODUÇÃO}

As últimas décadas assistiram a um interesse sem precedentes na área das Nanotecnologias e Nanociências, tratando-se atualmente de um dos domínios tecnológicos mais atrativos e de maior crescimento. A "revolução nano", comparada por muitos especialistas à revolução industrial do século XIX, promete produtos inovadores em áreas estratégicas como, por exemplo, a de energia, medicina, informação, segurança, ambiente e indústria.

A palestra de Richard Feynman na reunião anual da Sociedade Americana de Física, no Instituto de Tecnologia da Califórnia (Caltech), em 1959, é um marco histórico e científico ao antecipar desenvolvimentos em Nanotecnologia, sem que este termo tenha sido utilizado na ocasião. ${ }^{1}$ Nessa palestra, intitulada There's Plenty of Room at the Bottom, referiu-se à possibilidade de manipular a matéria à escala atómica e molecular e discutiram-se consequências em termos de progresso científico nesse novo contexto. As previsões de Feynman incluíram a miniaturização de dispositivos de armazenamento de informação e o desenvolvimento de microscópios com poder de resolução ao nível atómico. Contudo, a Nanotecnologia resulta efetivamente da sedimentação de conhecimento científico em áreas diversas, e para a qual contribuíram decisivamente avanços tecnológicos recentes, nomeadamente a descoberta e o aperfeiçoamento de microscópios que permitem a caracterização de nanoestruturas, métodos computacionais mais poderosos e novas técnicas de síntese em nanomateriais.

A invenção do microscópio de efeito túnel (Scanning Tunelling Microscope, STM), cuja patente é de 1980, deu a Gerd Binnig e Heinrich Rohrer o prémio Nobel da Física, em 1986. No STM é aplicada uma diferença de potencial entre a superfície da amostra e uma sonda muito fina que se aproxima da superfície do material a analisar, a uma distância atómica, originando uma corrente por efeito de túnel entre a ponta da sonda e a superfície. Uma modalidade de medição no STM faz com que a sonda seja deslocada ao longo da superfície (plano XY) de forma a varrer uma determinada área da amostra, mantendo a intensidade de corrente constante entre a ponta e a superfície. $\mathrm{O}$ ajuste da posição vertical (altura $\mathrm{Z}$ ) da ponta produz um mapa de contorno da superfície, que é tratado por computador para criar a imagem dos átomos (Figura 1). ${ }^{2}$ A manipulação individual de átomos foi amplamente divulgada em 1990, quando

*e-mail: tito@ua.pt cientistas da IBM manipularam 35 átomos de xénon utilizando um STM, movimentando um átomo de cada vez, de forma a "escrever" as iniciais I-B-M (Figura 1). ${ }^{2,3}$ A invenção do STM assenta em uma teoria científica poderosa inventada no século XX, a Teoria Quântica, abrindo caminho a uma nova revolução tecnológica para o século XXI, a Nanotecnologia.
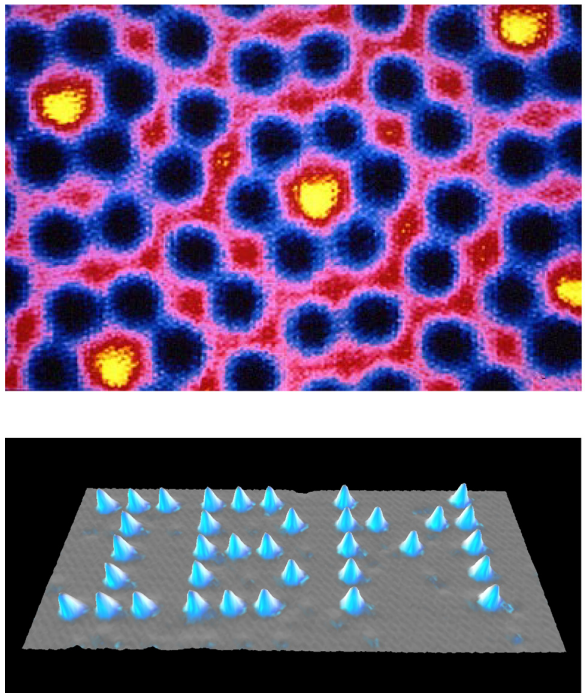

Figura 1. Uma das primeiras imagens obtidas por STM mostra átomos de silício com um aumento de 20 milhões de vezes. ${ }^{2}$ Cientistas no IBM descobriram como posicionar átomos individuais numa superfície metálica utilizando um microscópio de efeito túnel (STM). ${ }^{2}$ Reproduzidas da ref. 2 , com permissão da IBM

\section{NANOMATERIAIS E PROPRIEDADES DEPENDENTES DO TAMANHO}

Para além da possibilidade de miniaturização de dispositivos, os investigadores encontraram um "fabuloso mundo novo" em materiais com dimensões intermédias entre as das moléculas e as das partículas submicrométricas: os nanomateriais. Os nanomateriais apresentam propriedades distintas das propriedades das moléculas e dos sólidos cristalinos típicos, devido a efeitos de tamanho e de superfície que se tornam especialmente evidentes para dimensões tipicamente entre 1-100 nm. Existem inúmeros exemplos de estruturas 
de dimensões submicrométricas de origem natural, cujas dimensões são comparáveis às dimensões de materiais fabricados pelo homem. Os sistemas biológicos apresentam uma organização sistemática de estruturas e processos a uma escala nanométrica. O desenvolvimento de tecnologias que possibilitam a monitorização da interação de nanomateriais com estruturas biológicas tem impulsionado a criação de novas técnicas e dispositivos de diagnóstico e terapêutica. Os avanços tecnológicos nesta área levaram ao aparecimento de novos ramos de conhecimento como, por exemplo, a nanobiotecnologia e a nanomedicina.

Como consequência da diminuição do tamanho médio de partícula dos materiais, verifica-se um aumento da área superficial por volume (Figura 2). Por exemplo, a área superficial de um cubo de $1 \mathrm{~cm}$ de lado é de $6 \mathrm{~cm}^{2}$ para um volume de $1 \mathrm{~cm}^{3}$. Ao reduzir a aresta do cubo para $1 \mathrm{~mm}$, para um volume total de $1 \mathrm{~cm}^{3}$, existem 1000 cubos cuja área superficial totaliza $60 \mathrm{~cm}^{2}$. Se as dimensões forem expressas a uma escala nanométrica, isto é, admitindo que cada cubo tem $1 \mathrm{~nm}$ de lado, existem $10^{21}$ cubos por $1 \mathrm{~cm}^{3}$ de volume total, e a área superficial daí resultante é agora de $60.000 .000 \mathrm{~cm}^{2} !^{4}$ Analogamente, por exemplo, em uma nanopartícula (NP) de ferro com $30 \mathrm{~nm}$ de diâmetro, $5 \%$ dos átomos encontram-se à superfície, enquanto em uma NP de $10 \mathrm{~nm} 20 \%$ dos átomos encontram-se à superfície e já metade dos átomos do nanocristal são superficiais se for considerada uma NP de $3 \mathrm{~nm}$ de diâmetro. ${ }^{5}$ Ou seja, a percentagem de átomos à superfície, relativamente ao número total de átomos na partícula, aumenta quando o tamanho diminui. Este aumento significativo da área superficial, relativamente ao volume de material, determina diversas propriedades dos nanomateriais, sendo que a atividade catalítica é um exemplo sobejamente conhecido dos químicos.

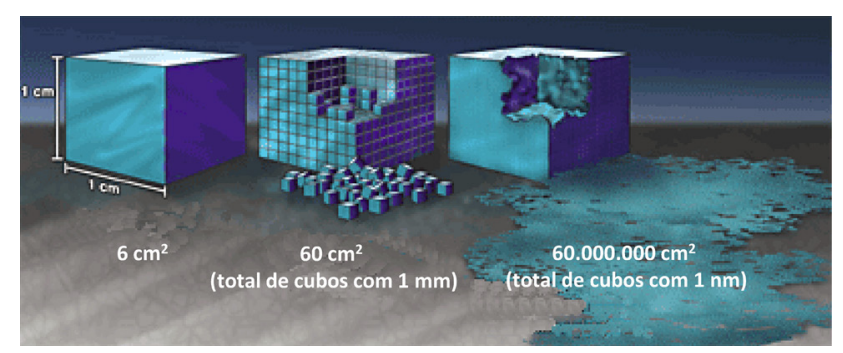

Figura 2. Evolução da área superficial com a diminuição das dimensões de um cubo. ${ }^{4}$ Reproduzida da ref. 4, com permissão de National Nanotechnology Initiative

Na próxima seção são indicados exemplos que ilustram o efeito de tamanho e de superfície nas propriedades óticas de NP metálicas, por exemplo, ouro e prata, de NP semicondutoras e nas propriedades magnéticas de NP ferromagnéticas. Tratam-se de sistemas que evidenciam a relevância das NP em termos de estudos fundamentais e cujas propriedades são ajustadas no processo de síntese química.

\section{NANOPARTÍCULAS DE SEMICONDUTORES - PONTOS QUÂNTICOS}

A pesquisa sistemática de efeitos quânticos de dimensão em partículas de semicondutores foi impulsionada por estudos desenvolvidos pelos grupos de Louis Brus, nos Estados Unidos, ${ }^{6}$ e de Alexander Efros, na antiga União Soviética. ${ }^{7}$ Estes investigadores observaram que as propriedades ópticas de materiais semicondutores, a partir de certas dimensões de partícula, eram dependentes do seu tamanho médio, tendo estabelecido uma base teórica para a compreensão dos efeitos quânticos de dimensão nestes materiais.

Estes efeitos de dimensão decorrem de alterações que se verificam na estrutura eletrónica de um determinado semicondutor quando o tamanho médio de partícula varia, tal como ilustra esquematicamente a Figura 3. A estrutura de níveis discretos de energia para átomos e moléculas tende para bandas de níveis de energia no caso de sólidos cristalinos convencionais. Entre estas duas situações limite, um material semicondutor tem um diagrama de níveis de energia dependente do número de átomos constituintes e, portanto, das dimensões dos cristais que o constituem. Como pode ser observado na Figura 3, o diagrama de energia de um nanocristal apresenta níveis discretos e o hiato ótico $\left(\mathrm{E}_{\mathrm{g}}\right)$ do semicondutor aumenta com a diminuição do tamanho de partícula. Trata-se de um efeito de dimensão de natureza quântica e por isso os nanocristais que exibem tais efeitos são designados de pontos quânticos (quantum dots, QD).

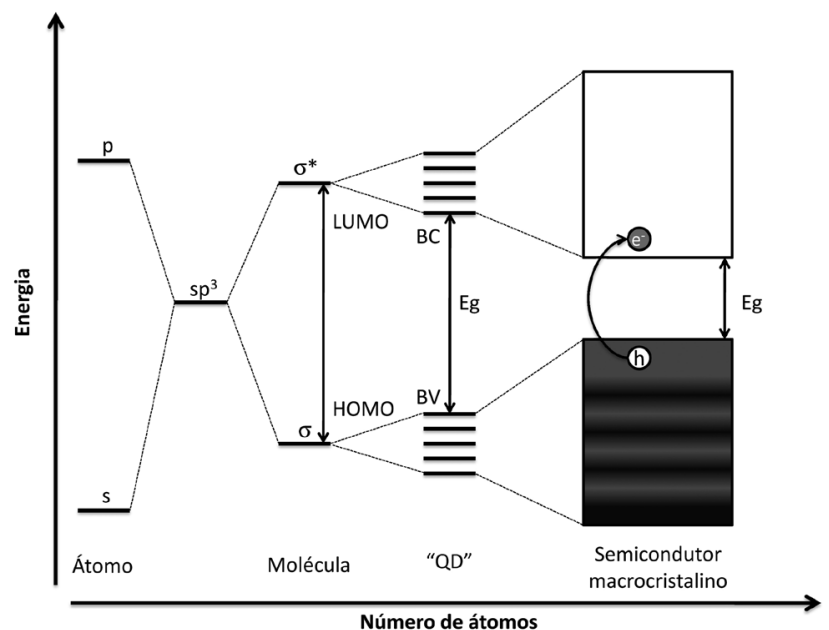

Figura 3. O diagrama de níveis de energia em um sólido cristalino depende do número de átomos combinados quimicamente. ${ }^{8}$ Os níveis de energia discretos das orbitais atómicas tendem para bandas de energia com o aumento do tamanho de partícula (na figura representado para um material semicondutor)

Quando num material semicondutor incide um fotão com energia para promover um eletrão da banda de valência (BV) para a banda de condução (BC), forma-se um par eletrão-lacuna na rede cristalina. Este par eletrão-lacuna é designado de excitão e pode ser descrito, em analogia a um sistema hidrogenoide, por um raio excitónico de Bohr $\left(r_{B}\right)$ que é característico para cada material semicondutor. Num material semicondutor macrocristalino, $r_{B}$ é significativamente inferior ao tamanho da partícula cristalina, daí resulta que o excitão é livre de migrar ao longo da estrutura. No entanto, quando as dimensões do cristal são reduzidas a tamanhos inferiores ou comparáveis a $r_{B,}$ as dimensões da partícula confinam a função de onda do excitão, aumentando a diferença energética (hiato ótico) entre a banda de valência e a banda de condução, devido à barreira de potencial de superfície. ${ }^{8}$

No início dos anos 1980, Efros e Brus estabeleceram expressões analíticas que evidenciam a dependência do hiato ótico de semicondutores em função do tamanho de partícula, quando este é menor ou comparável ao raio do excitão de Bohr (Equação 1). O hiato ótico $E_{n}$ aumenta relativamente ao valor do material macrocristalino $\left(E_{g}\right)$ de um termo de confinamento energético cuja dependência com o raio do cristal é de $1 / R^{2}$.

$$
E_{n}=E_{g}+\frac{h^{2} \pi^{2}}{2 \mu R^{2}}
$$

onde $E_{g}$ é o valor do hiato de energia para o material semicondutor macrocristalino; $h$ é a constante de Planck; $R$ é o raio da partícula e $\mu$ é 
a massa reduzida do excitão dada por: $\mu=m_{e}^{*} \times m_{h}^{*} /\left(m_{e}^{*}+m_{h}^{*}\right)$ em que $m_{e}^{*}$ e $m_{h}^{*}$ são as massas efetivas do eletrão e lacuna, respetivamente. ${ }^{8}$

Em outra expressão, para além do termo relativo ao confinamento quântico é incluído um termo relativo à interação de Coulomb (Equação 2):

$$
\Delta E=\frac{h^{2} \pi^{2}}{2 \mu R^{2}}-\frac{1.8 e^{2}}{\varepsilon R}
$$

em que $e$ é a carga elementar e $\varepsilon$ é a constante dielétrica do semicondutor macrocristalino.

Embora a Equação 2 resulte de um modelo simplificado, permite avaliar o comportamento quântico de nanopartículas (NPs) semicondutoras, devido a efeitos de tamanho de partícula. Por exemplo, esta equação é particularmente útil em síntese química para se obter uma estimativa de tamanho médio de partículas esferoides pela análise dos espetros eletrônicos experimentais.

Uma consequência importante da Equação 2 é a possibilidade de se ajustar a absorção (ou emissão) de luz de um dispositivo semicondutor, não só através da composição química do semicondutor mas, também, manipulando o tamanho médio de partícula. Um material exemplar deste comportamento é o CdSe. O CdSe macrocristalino apresenta uma cor castanha, enquanto para NP de CdSe com cerca de $2 \mathrm{~nm}$ até pouco mais de $8 \mathrm{~nm}$ de diâmetro, devido a efeitos de confinamento quântico, observam-se cores que vão do amarelo ao vermelho, sob luz branca, e do azul ao vermelho, sob luz UV, dependendo do tamanho médio de partícula (Figura 4). ${ }^{9}$ A cor observada por emissão de fotões no visível deve-se à recombinação de pares eletrão-lacuna, sendo por isso designada por emissão excitónica.
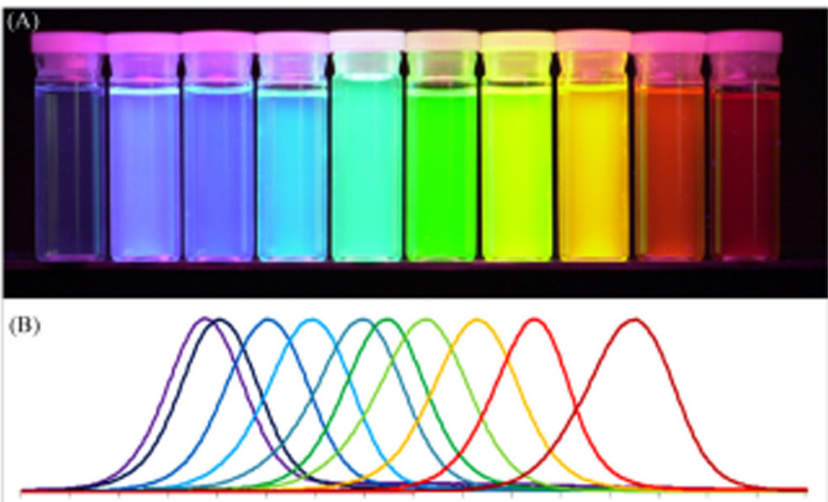

$\begin{array}{lllllllllllllllll}400 & 420 & 440 & 460 & 490 & 500 & 520 & 540 & 560 & 592 & 600 & 620 & 640 & 660 & 692 & 700 & 720\end{array}$

Figura 4. A "cor" da emissão dos QD de CdSe pode ser afinada de forma a varrer o espectro do visível. (A) apresentam-se coloides de QD de CdSe (revestidos com $\mathrm{ZnS}$ ) com diferentes tamanhos médios (de cerca de 2 a pouco mais de $8 \mathrm{~nm})$ e (B) os respectivos espectros de fotoluminescência. Adaptada da ref. 9, com permissão da Elsevier

A emissão por fotoluminescência em QD é influenciada pela natureza da sua superfície. Em materiais nanocristalinos a percentagem de defeitos superficiais, por exemplo átomos com valência incompleta ou falhas atómicas, é relativamente elevada e originam estados de energia ("armadilhas") que capturam os transportadores de carga, reduzindo a probabilidade de recombinação excitónica e aumentando a possibilidade de ocorrerem eventos não radiativos. A recombinação excitónica é maximizada por passivação dos defeitos superficiais, pelo recurso a bases de Lewis que estabelecem ligações com sítios na superfície e/ou por crescimento de uma coroa inorgânica de um material semicondutor de hiato ótico largo (Figura 5). Esta coroa inorgânica cria um poço de energia potencial, concentrando os transportadores de carga na partícula interna. ${ }^{10,11}$ Desta forma, o efeito dos estados de superfície na eficiência de emissão terá um impacto reduzido. Na forma coloidal é possível revestir QD de CdSe com coroas de materiais semicondutores com $\mathrm{E}_{\mathrm{g}}$ superior como, por exemplo, o $\mathrm{ZnS}$ ou $\mathrm{CdS}$. Estes materiais não só isolam o núcleo de $\mathrm{CdSe}$ como o protegem relativamente à oxidação em condições ambiente, já que o ião $S^{2-}$ tem um potencial de redução maior do que o $\mathrm{Se}^{2-}$, reduzindo assim a degradação foto-oxidativa da superfície.
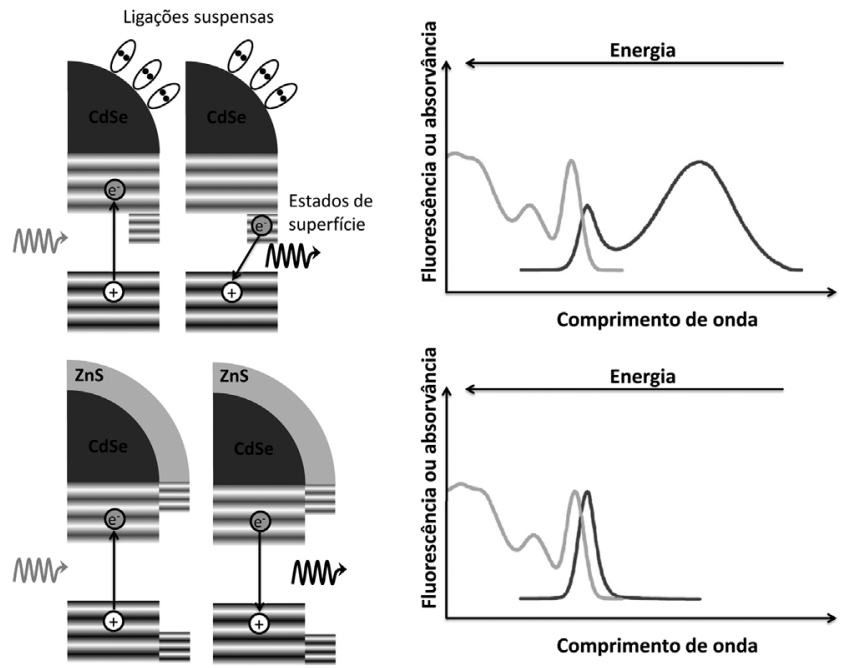

Figura 5. As propriedades ópticas de QD são influenciadas pelos estados de superfície. Adaptada da ref. 11, com permissão da American Chemical Society

\section{NANOPARTÍCULAS METÁLICAS}

A utilização de partículas de ouro na forma coloidal pode ser seguida até ao século V A.C. no Egito e China e, desde então, são vários os exemplos da sua utilização com motivações decorativas ou medicinais. ${ }^{12}$ Um exemplo no uso de ouro nanocristalino para fins decorativos que se tornou famoso com o advento de investigação em nanomateriais é o do cálice de Lycurgus (Figura 6). Trata-se de um cálice datado do período romano (século IV, D.C.) fabricado em matriz vítrea e cuja cor depende do ângulo de incidência da luz visível sobre a sua superfície. A cor verde resulta da reflexão da luz pela superfície do cálice, enquanto a luz ao ser transmitida através do vidro origina uma cor vermelha. Este efeito ótico deve-se à existência de nanopartículas metálicas, nomeadamente de ouro e prata, que se encontram dispersas no vidro do cálice, originando um vidro dicroico.
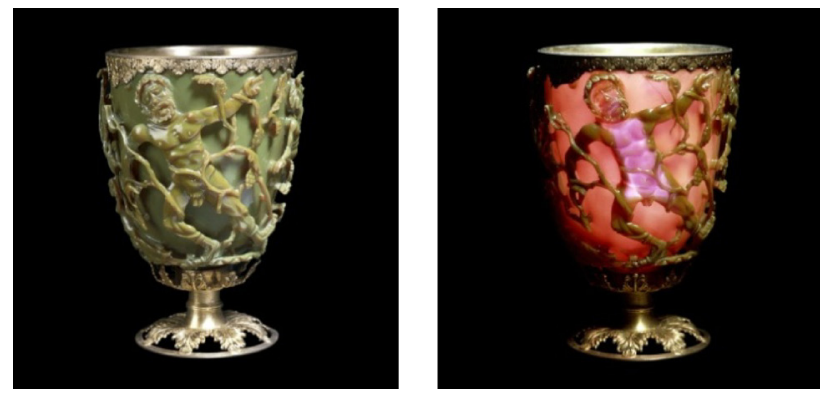

Figura 6. Fotografias do cálice de Lycurgus em exposição no British Museum em Londres. $O$ vidro apresenta uma cor verde em condições normais de iluminação, mas apresenta uma cor vermelha quando o cálice é iluminado a partir do interior. Reproduzida com permissão de ${ }^{\circledR}$ Trustees of the British Museum

Em 1857, Michael Faraday adiantou uma primeira explicação científica para a cor vermelha exibida por coloides de Au preparados 
a partir de um sal de ouro (III), posteriormente reduzido com fósforo num sistema bifásico água e $\mathrm{CS}_{2} \cdot{ }^{13}$ Faraday sugeriu que a interação da luz visível com partículas de ouro finamente divididas dispersas no solvente estaria na origem das cores observadas. ${ }^{14}$ Quase 100 anos depois, Turkevich ${ }^{15}$ utilizou um microscópio eletrónico para revelar que os coloides de cor rubi, preparados por métodos de síntese similares, consistiam em nanopartículas de ouro com tamanho médio de $6 \pm 2 \mathrm{~nm} \cdot{ }^{14-16}$ Os estudos de G. Mie permitiram a resolução das equações de Maxwell para a absorção e dispersão da radiação eletromagnética por esferas de pequenas dimensões e constituem a base teórica para o estudo da interação da radiação com estas pequenas partículas. ${ }^{17}$

A propagação de ondas eletromagnéticas ao longo da interface entre um metal e o meio dieléctrico circundante cria um campo eletromagnético acoplado à oscilação coerente dos eletrões da banda de condução. As energias destas oscilações encontram-se quantizadas e os respetivos quanta de energia designam-se por plasmões de superfície (SP). Para partículas metálicas com um diâmetro muito inferior ao comprimento de onda da radiação incidente $d<<\lambda$, o campo elétrico induz a polarização de cargas, com os eletrões livres na partícula deslocados relativamente à rede catiônica do metal. A diferença na distribuição de cargas à superfície da partícula origina uma força restauradora, verificando-se condições de ressonância quando a oscilação dos eletrões livres está em fase com a radiação eletromagnética, isto é, para o valor de frequência de ressonância do plasmão de superfície (SPR, Surface Plasmon Resonance).$^{18}$ As condições de ressonância dependem das propriedades dielétricas e da densidade de eletrões livres dos metais, podendo ser modeladas utilizando as equações de Mie. Os coloides de cobre, ouro e prata têm a frequência de SPR na parte visível do espetro eletromagnético, o que tem motivado muitos estudos de efeitos plasmónicos nestes metais. ${ }^{19}$

A cor de coloides de NP de ouro de forma aproximadamente esférica pode ir desde o azul, passando por vários tons de vermelho até ao laranja, quando o tamanho de partícula é reduzido até cerca de $3 \mathrm{~nm} .{ }^{18}$ Para nanopartículas de tamanhos mais reduzidos, o formalismo de Mie tem de ser alterado de modo a incorporar efeitos quânticos de dimensão. Em partículas alongadas (elipsoides e bastonetes) surgem duas bandas plasmónicas no espetro eletrónico, estando relacionadas com os modos de oscilação transversal e longitudinal. As variações observadas podem ser significativas; por exemplo, calcula-se que para partículas de forma esférica, um aumento no diâmetro médio de 10 a $100 \mathrm{~nm}$ origina um desvio para o vermelho de $47 \mathrm{~nm}$, (Figura 7a) enquanto para partículas elipsoidais, um aumento na razão de aspeto de 2,5 a 3,5 origina um desvio batocrómico de $92 \mathrm{~nm}$ no máximo de absorção da banda plasmónica do modo longitudinal. ${ }^{19}$

A resposta ótica de nanopartículas metálicas (ouro) depende também de outros parâmetros, tais como a função dielétrica do meio circundante (Figura 7b) ${ }^{20,21}$ e a distância entre nanopartículas vizinhas que, por sua vez, determina o acoplamento plasmónico entre partículas. ${ }^{22,23} \mathrm{~A}$ variação concertada destes parâmetros permite o ajuste das propriedades óticas de nanoestruturas de ouro. Mais especificamente, está estabelecido que a posição espetral da ressonância SP é desviada para maiores comprimentos de onda quando é aumentada a constante dielétrica do meio circundante. Isto se deve ao enfraquecimento da força restauradora, devido à formação de cargas de polarização na interface com o dielétrico. ${ }^{21}$ Para além disso, quando as NP de ouro se aproximam, para distâncias comparáveis com o seu diâmetro, a banda SPR característica da NP isolada começa a desviar-se para o vermelho e apresenta-se alargada, como resultado do acoplamento plasmónico. $^{23}$

\section{NANOPARTÍCULAS MAGNÉTICAS}

Os materiais reagem ao efeito de aplicação de um campo a

\section{Tamanho}

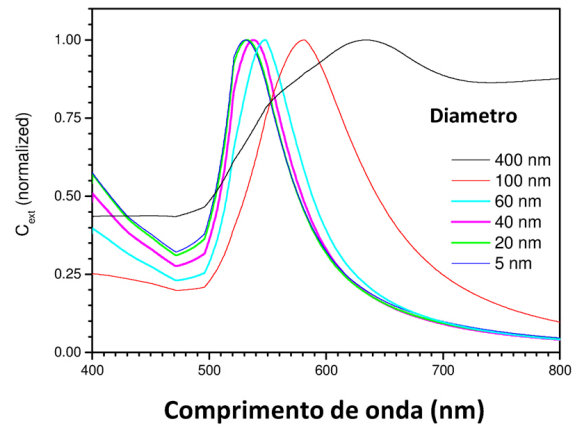

b

\section{Índice de refracção do meio}

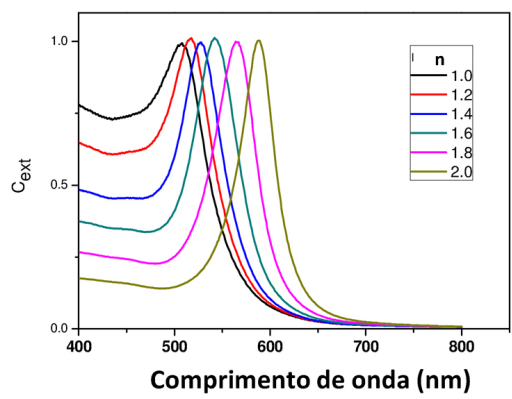

Figura 7. Espectros óticos de NP coloidais de Au evidenciando alguns parâmetros que afetam a frequência de ressonância plasmónica: (a) diâmetro das $N P e(b)$ índice de refração do meio em que as NP se encontram

magnético externo, podendo ser classificados consoante a interação resultante é atrativa ou repulsiva. A natureza destas forças atrativas ou repulsivas pode ser descrita em termos da formação de dipolos magnéticos, podendo estes ser entendidos, em termos simples, como minúsculas barras magnéticas que possuem polos opostos. O material designa-se diamagnético quando não tem dipolos magnéticos na ausência de um campo magnético externo mas pela sua aplicação surgem dipolos magnéticos fracos. A magnetização de um material diamagnético ocorre na direção oposta à do campo, resultando assim numa força repulsiva fraca. Exemplos de substâncias e materiais diamagnéticos são a água e a maioria dos polímeros orgânicos. O material é designado paramagnético quando apresenta dipolos magnéticos orientados aleatoriamente mas que podem ser alinhados por ação de um campo magnético externo (Figura 8). A magnetização do material paramagnético ocorre na mesma direção do campo externo. Estes materiais se caracterizam, ainda, por uma fraca interação entre os dipolos. Tipicamente a curva de magnetização destes materiais traduz uma variação linear da suscetibilidade magnética inversamente proporcional à temperatura (lei de Curie). Entre os materiais paramagnéticos encontram-se, por exemplo, metais alcalinos e alcalino-terrosos, e metais de transição (como a platina).

Em um material ferromagnético, os dipolos magnéticos existem mesmo na ausência de um campo magnético externo e exercem um efeito de longo alcance, exibindo um momento magnético permanente. Os materiais ferromagnéticos são caracterizados por uma interação de troca, comportamento de histerese e coercibilidade finita, Hc. Na curva de magnetização típica (Figura 8), a magnetização encontra-se sempre defasada do campo magnético aplicado, o que origina um ciclo de histerese devido à presença de domínios magnéticos no material. 

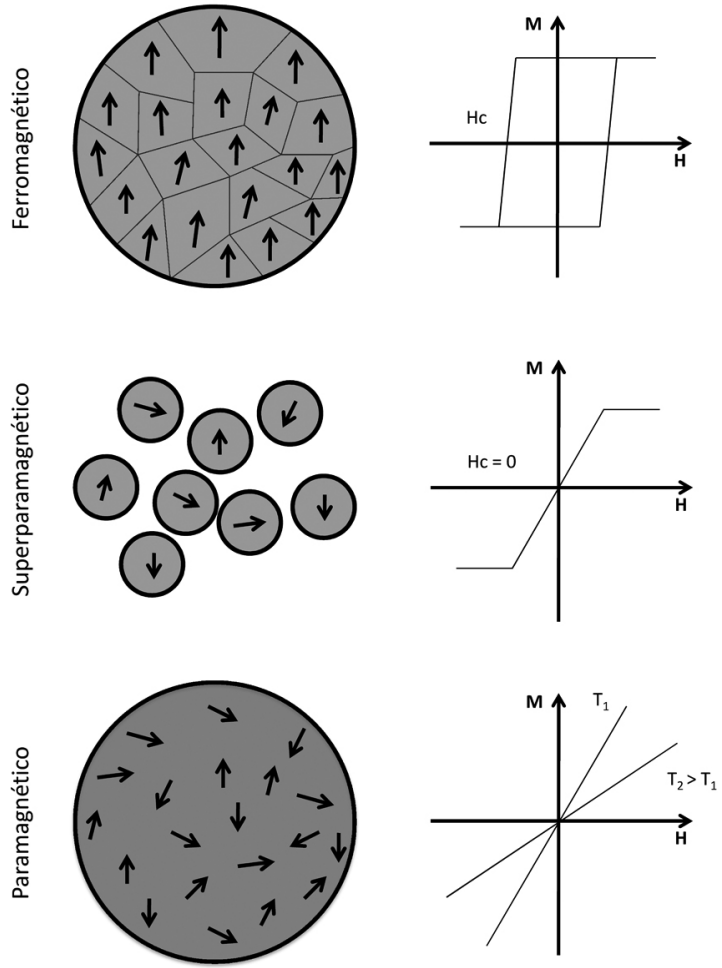

Figura 8. Diferentes tipos de comportamento magnético em materiais

Da análise da curva de magnetização é possível obter o valor da magnetização de saturação, Ms, ou o valor máximo de $\mathrm{M}$, a magnetização remanescente, $\mathrm{Mr}$, ou a magnetização residual na ausência de campo, a coercibilidade, Hc, ou o valor do campo externo que é necessário para reduzir a magnetização de volta a zero. Exemplos de materiais ferromagnéticos são os metais de transição $\mathrm{Fe}$, Co e Ni, assim como as ligas metálicas, $\mathrm{CoPt}$, $\mathrm{CoPt}_{3}, \mathrm{FePt}, \mathrm{FePt}_{3}, \mathrm{FeCo}$.

A diferença na origem do momento magnético permanente pode também ser usada para distinguir ferromagnetismo de ferrimagnetismo e de antiferrimagnetismo. No material ferrimagnético existem sempre dipolos magnéticos mais fracos alinhados antiparalelamente com os dipolos magnéticos mais fortes adjacentes, na ausência de campo magnético externo. Exemplos de materiais ferrimagnéticos são os óxidos de ferro: magnetite, $\mathrm{Fe}_{3} \mathrm{O}_{4}$ e maguemite, $\gamma-\mathrm{Fe}_{2} \mathrm{O}_{3}$ e as ferrites $\mathrm{MFe}_{2} \mathrm{O}_{3}$ em que $\mathrm{M}=\mathrm{Co}, \mathrm{Ni}, \mathrm{Co}, \mathrm{Mn}$, etc. Para um material antiferromagnético, os dipolos adjacentes são antiparalelos, na ausência de um campo externo, cancelando-se mutuamente. Os óxidos metálicos $\mathrm{MnO}, \mathrm{CoO}$ e $\mathrm{NiO}$ são exemplos de materiais antiferromagnéticos. Em geral, os materiais apelidados trivialmente de magnéticos são aqueles que apresentam características de ferroou ferrimagnetismo. ${ }^{24}$

Com a diminuição do tamanho de partícula abaixo de certo valor crítico, a formação de domínios torna-se energeticamente desfavorável e o domínio magnético pode coincidir com a NP magnética. Este tamanho crítico é característico da composição do material. Em uma partícula monodomínio, a mudança na orientação da magnetização ocorre através de uma rotação coerente dos spins, o que origina uma coercibilidade relativamente elevada. ${ }^{5}$ Se o tamanho de partícula do material ferromagnético for ainda mais reduzido, a energia de agitação térmica $\left(K_{B} T\right)$ é suficiente para desalinhar a orientação da componente da magnetização, de tal forma que na ausência de um campo magnético externo a magnetização resultante é nula. Estes materiais não apresentam coercibilidade e comportam-se como paramagnetes com um momento magnético clássico elevado, sendo que as nanopartículas são designadas por superparamagnéticas.

\section{SÍNTESE QUÍMICA DE NP COLOIDAIS}

\section{Aspetos fundamentais}

A preparação de partículas com um controle apertado do tamanho e/ou morfologia uniforme é conseguida através da interação concertada de átomos ou moléculas durante o processo de síntese, que ocorre geralmente em fase vapor ou líquida. A formação de partículas coloidais em fase líquida a partir de soluções homogéneas é especialmente interessante pois as variáveis experimentais podem ser ajustadas na bancada do químico, nomeadamente a concentração de reagentes, temperatura, $\mathrm{pH}$ (em soluções aquosas), presença de aditivos (tensioativos, sais, polímeros, etc.), propriedades do solvente (temperatura de ebulição, afinidade com as superfícies criadas), adição de sementes de nucleação, entre outros. A influência destes fatores durante os processos de nucleação e crescimento das partículas ditarão o tamanho e/ou morfologia final das partículas.

De acordo com a teoria clássica, a nucleação em solução homogênea engloba um conjunto de eventos que resultam na formação de um núcleo de tamanho crítico da nova fase sólida. A variação da energia livre total relacionada com este processo reflete o balanço entre duas contribuições: uma contribuição negativa da variação do potencial químico, relacionada com a formação de uma fase sólida em meio líquido (energia de volume) e uma contribuição positiva, devida à criação de uma nova superfície (energia de superfície):

$$
\Delta G=4 \pi r^{2} \gamma+\frac{4}{3} \pi r^{3} \Delta G_{v}
$$

sendo $\gamma$ a energia livre de superfície por unidade de área e tem sempre um valor positivo; $\Delta G_{v}$ a variação de energia livre do sólido por unidade de volume e é de valor negativo, desde que a solução se encontre em condições de sobressaturação (Equação 4):

$$
\Delta G_{v}=-R T \ln S / V_{m}
$$

Como resultado deste balanço, a variação da energia livre total $(\Delta \mathrm{G})$ atinge um máximo, $\Delta G^{*}$, para certo valor do raio do núcleo, designado por raio crítico, $r^{*}$ (Figura 9). Para um valor do raio superior a $r^{*}$, o crescimento da partícula é acompanhado por uma diminuição da energia livre total e por isso o processo ocorre espontaneamente; quando o núcleo é menor do que $r^{*}$, o seu crescimento implica um aumento de energia livre total e a tendência é que o núcleo se dissolva. Pode-se determinar o valor do raio crítico considerando $d \Delta G / d r=0$ :

$$
r^{*}=\frac{-2 \gamma}{\Delta G_{v}}=\frac{2 \gamma V_{m}}{R T \ln S}
$$

No processo de nucleação homogênea, a sobressaturação $(\mathrm{S})$ deverá ser suficientemente elevada de forma que o raio crítico seja menor do que o tamanho dos núcleos primários formados. ${ }^{25,26}$ Por outro lado, o valor do raio crítico acompanha o valor da sobressaturação ao longo de todo o processo de formação das partículas, pelo que o valor de $S$ determina os fenómenos de nucleação e crescimento.

Uma vez que os núcleos primários são muito pequenos, e devido ao movimento Browniano, há a probabilidade de se ultrapassar a barreira de energia de Gibbs, o que ditará a sobrevivência de alguns dos núcleos primários e o seu crescimento posterior. Enquanto a nucleação determina o número de partículas e, em grande medida, o seu tamanho médio final, a cinética de crescimento por sua vez vai determinar a velocidade de consumo do material existente e as condições de sobressaturação. A obtenção de partículas morfologicamente uniformes depende do controle rigoroso do processo de crescimento, que pode envolver mecanismos diversos. 


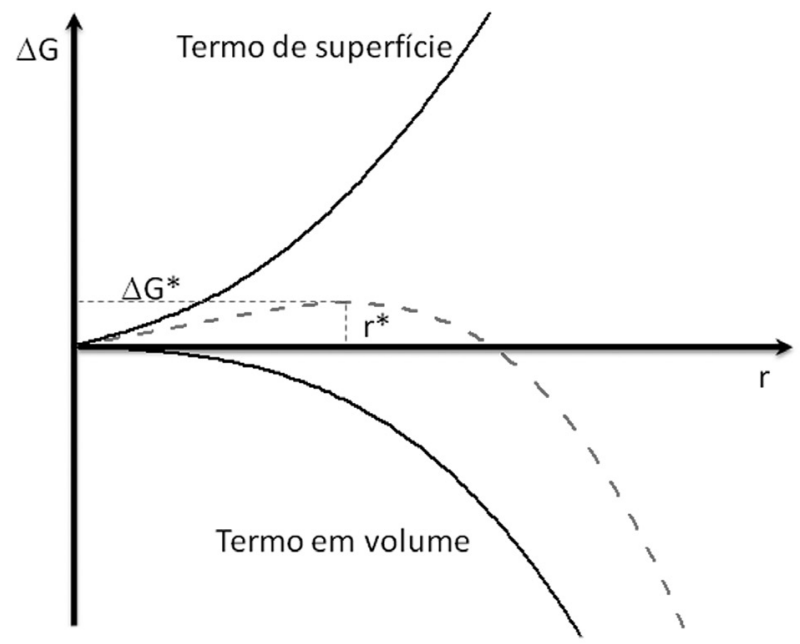

Figura 9. Esquema representativo da variação da energia livre total em função do tamanho de partícula primária

A investigação dos mecanismos de precipitação de partículas coloidais com uma distribuição de tamanhos estreita (amostra "monodispersa") data dos anos 1940, quando LaMer e Dinegar introduziram o conceito de nucleação instantânea (burst nucleation). Neste processo, forma-se um número elevado de núcleos primários em um intervalo de tempo curto, que crescem depois sem que ocorram nucleações posteriores. Como a formação de núcleos ocorre de forma quase simultânea, o histórico de crescimento é praticamente o mesmo, desde que devidamente controlado, permitindo obter amostras com uma distribuição estreita de tamanhos de partícula. ${ }^{27}$

A síntese de nanopartículas inorgânicas com elevado grau de monodispersidade tem sido realizada recorrendo-se frequentemente a solventes de elevado ponto de ebulição, que normalmente também atuam como agentes tensioativos. Neste caso, há dois métodos principais para se criar condições de nucleação instantânea - a injeção dos reagentes no solvente que se encontra à temperatura elevada: injeção a quente ${ }^{28,29}$ ou pelo aquecimento conjunto do sistema reacional e solvente - aquecimento em lote. ${ }^{30-33}$

Bawendi e colaboradores descreveram uma técnica de injeção de grande sucesso, em artigo que é atualmente uma referência incontornável na síntese de nanomateriais, sobre a preparação de nanopartículas de $\mathrm{CdE}(\mathrm{E}=\mathrm{S}, \mathrm{Se}, \mathrm{Te}) .{ }^{34} \mathrm{O}$ grau elevado de sobressaturação de espécies precursoras é conseguido pela injeção rápida dos reagentes num solvente de elevado ponto de ebulição, a uma temperatura que permite a decomposição dos reagentes. Resulta, assim, uma nucleação instantânea de $\mathrm{CdE}$ de forma a reduzir a energia livre resultante do aumento rápido da sobressaturação. No decorrer da nucleação, a concentração de precursor em solução diminui, bem como a velocidade de nucleação. Por outro lado, a injeção de uma solução a uma temperatura inferior à temperatura a que se encontra o solvente leva à sua diminuição quase imediata, que no caso de ser uma diminuição significativa trava o processo de nucleação. O processo de crescimento posterior ocorre de forma concertada pelos núcleos primários formados, que irão consumir os reagentes existentes em solução. Este tem sido o método de eleição para a preparação de nanocristais de qualidade elevada nomeadamente de semicondutores. ${ }^{28,34}$

O método de aquecimento em lote é um processo em que a mistura de reagentes e solventes é feita geralmente à temperatura ambiente, e só depois é aquecida até uma temperatura superior para que ocorra a reação de formação do sólido. Este método, pela sua simplicidade, é particularmente vantajoso para produção em larga escala. Embora seja um processo mais simples, a uniformidade de tamanhos das nanopartículas formadas é, em alguns casos, comparável aos resultados obtidos para o método de injeção. ${ }^{30,31}$ Por exemplo, estudos realizados na preparação de NP de óxidos de ferro, a partir da decomposição térmica de oleato de ferro, demonstraram que o mecanismo de aquecimento em lote segue uma cinética semelhante ao proposto por LaMer. A decomposição do oleato de ferro para a formação dos óxidos de ferro passa pela formação de espécies intermediárias de ferro. Estas espécies intermediárias, e não o oleato de ferro, servem de precursores da formação do óxido de ferro e contribuem para o grau de sobressaturação. Estes precursores irão acumular-se no meio reacional até que o nível de sobressaturação atinja um valor crítico, que seja suficiente para ultrapassar a barreira da energia livre de Gibbs. Após o sistema atingir este grau de sobressaturação crítico, a nucleação ocorre de forma semelhante à descrita para o mecanismo de injeção a quente. ${ }^{35}$

Se o processo de nucleação instantânea permitiu a formação de um elevado número de núcleos primários de dimensões reduzidas (ainda assim superiores ao raio crítico, $r^{*}$ ), por diminuição da sobressaturação e, por consequência da energia livre, as condições de sobressaturação existentes e a elevada energia superficial destes núcleos primários são suficientes para que o processo de crescimento das partículas ocorra de forma a atingirem maiores dimensões. As NP formadas possuem uma elevada energia de superfície e têm tendência para continuar a crescer. Quando as espécies precursoras do sólido se encontram praticamente consumidas, as nanopartículas ainda podem diminuir a sua energia de superfície através de um processo de crescimento secundário, que é um mecanismo de crescimento por agregação. De forma a obter nanopartículas individualizadas, este processo deverá ser inibido pela adição de agentes tensioativos que, ao funcionalizarem a superfície das nanopartículas, previnem a sua agregação. O revestimento da superfície de NP com tensioativos permite não só o controle da velocidade de crescimento das NP, como também impede o seu crescimento por agregação. A escolha adequada dos tensioativos (pode ou não coincidir com o solvente) determina a estabilidade coloidal das NP e permite controlar sua morfologia.

\section{Síntese de NP semicondutoras - QD}

Os primeiros métodos de preparação de soluções coloidais de QD não permitiam a preparação de QD de elevada qualidade, no que diz respeito à sua cristalinidade e distribuição estreita de tamanhos, em quantidades razoáveis. A literatura oferece vários artigos de revisão sobre a síntese de $\mathrm{QD}^{36}$ onde este tópico é abordado e aqui serão focados exemplos de síntese de QD de materiais dos grupos II/VI, onde os conceitos abordados na secção anterior se encontram presentes. O trabalho de Murray, Norris e Bawendi demonstrou ser possível a preparação de QD de qualidade elevada em quantidades razoáveis. ${ }^{34}$ Estes autores descreveram a síntese de nanocristais relativamente monodispersos (dispersão inferior a 5\%) de $\mathrm{CdE}(\mathrm{E}=\mathrm{S}, \mathrm{Se}, \mathrm{Te})$, pela injeção de dimetilo de cádmio $\left(\mathrm{Cd}\left(\mathrm{CH}_{3}\right)_{2}\right)$ e uma fonte do calcogeneto respetivo (TOPE) num solvente à temperatura elevada (tipicamente entre $200-300{ }^{\circ} \mathrm{C}$ ), o que resulta num processo de nucleação instantânea. Esta nucleação rápida reduz a concentração de reagentes e limita a probabilidade de ocorrência de novos episódios de nucleação.

A utilização de temperaturas de síntese elevadas requer solventes surfactantes compatíveis como, por exemplo, o óxido de trioctilfosfina (TOPO). As moléculas de TOPO se coordenam à superfície dos nanocristais formados, formando uma camada hidrofóbica superficial que atua como barreira à coalescência das NP. Atualmente são empregues muitos outros solventes surfactantes com características similares como, por exemplo, a oleilamina, trioctilamina e ácido oleíco. O tamanho dos nanocristais pode ser ajustado pelo controle experimental de diversos parâmetros como tipo de reagente, temperatura e tempo de reação, aumentando o tamanho médio das partículas com o aumento 
de temperatura. Ainda a velocidade de injeção, temperatura de injeção e injeções de precursores posteriores à mistura reacional permitem o controle do tamanho, morfologia e do próprio polimorfo obtido. ${ }^{37}$

O método utilizando TOPO foi adaptado com sucesso no revestimento de núcleos de QD com uma capa de $\mathrm{ZnS}$, um material semicondutor de hiato ótico maior. Este processo requer a injeção de soluções de dimetilo ou dietilo de zinco, e de $\left[\left(\mathrm{CH}_{3}\right)_{3} \mathrm{Si}\right]_{2} \mathrm{~S}$, no solvente que contém NP de CdE que servem de núcleos para crescimento da fase $\mathrm{ZnS} .{ }^{11}$ A toxicidade, manipulação e custos dos materiais de partida tornam este método desvantajoso relativamente a alternativas descritas posteriormente.

A Figura 10 ilustra a anatomia de um QD, composto por um núcleo do material semicondutor oticamente ativo, revestido por uma camada de um semicondutor de hiato ótico superior e por moléculas surfactantes coordenadas à superfície da nanopartícula.

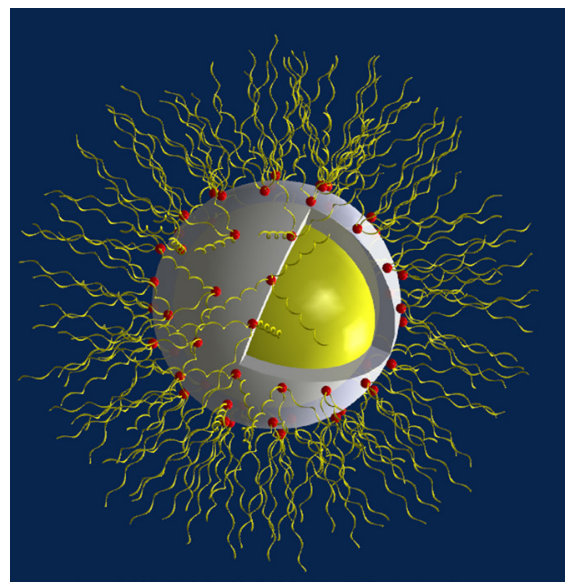

Figura 10. Anatomia de um ponto quântico $(Q D)$

Embora mantendo o conceito subjacente ao método original no que diz respeito ao controle da nucleação e crescimento em solventes tensioativos, ${ }^{34}$ surgiram posteriormente métodos alternativos. Uma das propostas avançada por Trindade e O'Brien envolve a decomposição térmica de precursores unimoleculares, isto é, um composto de coordenação contendo ambos os elementos necessários à formação do material semicondutor, tais como complexos metálicos do tipo ditiocarbamato ou disselenocarbamato. ${ }^{38,39}$ Este método é particularmente interessante na utilização de precursores estáveis ao ar e permite, em princípio, a síntese de uma variedade de semicondutores num processo de uma só etapa. ${ }^{39,40}$ Este método tem sido também investigado para revestir QD de CdSe com ZnS ou CdS, quer por decomposição térmica do complexo de ditiocarbamato do respectivo metal ${ }^{41}$ ou por sonólise de xantatos metálicos. ${ }^{42}$

Um avanço importante na síntese de QD por adaptação do método TOPO original foi feito por Peng e Peng, no decorrer do estudo do mecanismo de formação de nanocristais semicondutores ${ }^{46}$ Estes investigadores observaram que a introdução de um ligante que se coordene fortemente ao metal na mistura reacional, como é o caso dos ácidos fosfónico, hexilfosfónico (HPA) ou tetradecilfosfónico (TDPA), o $\mathrm{Cd}\left(\mathrm{CH}_{3}\right)_{2}$ converte-se rapidamente num complexo de Cd(II) com HPA/TDPA, que funciona como precursor contendo cádmio. Estes complexos podem ser igualmente obtidos por dissolução de outros compostos de $\mathrm{Cd}(\mathrm{II})$, tais como $\mathrm{CdO}$ ou $\mathrm{Cd}\left(\mathrm{CH}_{3} \mathrm{CO}_{2}\right)_{2}$. Como estes são compostos de cádmio disponíveis comercialmente e fáceis de manipular em condições ambiente, a produção de nanocristais de CdE a uma maior escala e com menor custo é facilitada, por exemplo, fazendo reagir diretamente com o não metal:

$$
\mathrm{Cd}\left(\mathrm{CH}_{3} \mathrm{CO}_{2}\right)_{2}+\mathrm{Se} \stackrel{\mathrm{TOP} / \mathrm{TOPO}, \Delta}{\longrightarrow} \mathrm{CdSe}+\ldots
$$

Por outro lado, é possível realizar-se a síntese em outros solventes orgânicos, como ácidos carboxílicos e aminas de cadeia carbonada longa. ${ }^{47}$

Atualmente, a síntese de NP utilizando solventes tensioativos de elevado ponto de ebulição é a via mais comum de preparação de NP de QD do grupo II/VI (Tabela 1). Embora as NP estabilizadas com tensioativos sejam de qualidade elevada, existe um grande interesse em desenvolver estratégias de síntese de QD com qualidade comparável mas em meio aquoso. Como exemplos incluem-se os QD de $\mathrm{HgTe}$ e $\mathrm{CdTe},{ }^{48}$ e a síntese em solução aquosa de nanocristais de $\mathrm{CdS},{ }^{25}$

Tabela 1. Métodos comuns de síntese de QDs

\begin{tabular}{|c|c|c|c|c|c|}
\hline Material & Precursores & surfactantes & solventes & método & Ref. \\
\hline $\mathrm{ZnS}$ & $\mathrm{ZnEt}_{2}, \mathrm{~S}$ & HDA, OAm, OAc & ODE & DT & 43 \\
\hline \multirow[t]{4}{*}{$\mathrm{CdS}$} & $\mathrm{CdMe}_{2},(\mathrm{TMS})_{2} \mathrm{~S}$ & TOP, TOPO & & DT & 34 \\
\hline & $\mathrm{CdO}, \mathrm{S}$ & OAc & ODE & DT & 44 \\
\hline & Xantato de Cádmio & HDA & & DT & 45 \\
\hline & Carbamato de Cádmio & TOP, TOPO & TOPO & DT & 39 \\
\hline \multirow[t]{5}{*}{$\mathrm{CdSe}$} & $\mathrm{CdMe}_{2}$, TOPSe & TOPO & TOPO & DT & \\
\hline & {$\left[\left(\mathrm{CH}_{3}\right) \mathrm{CdSe}_{2} \mathrm{CN}\left(\mathrm{C}_{2} \mathrm{H}_{5}\right)_{2}\right]_{2}$} & TOPO/TOP & & DT & 38 \\
\hline & {$\left[\mathrm{Cd}\left(\mathrm{S}_{2} \mathrm{CN}\left(\mathrm{C}_{2} \mathrm{H}_{5}\right)_{2}\right)_{2}\right]_{2}$} & TOPO/TOP & & DT & 39 \\
\hline & {$\left[\mathrm{Cd}\left(\mathrm{Se}_{2} \mathrm{CN}\left(\mathrm{C}_{2} \mathrm{H}_{5}\right)_{2}\right)_{2}\right]_{2}$} & & & DT & 39 \\
\hline & $\mathrm{Cd}(\text { xantato })_{2}$ & TOPO/TOP & & DT & 40 \\
\hline CdTe & $\mathrm{CdMe}_{2},(\mathrm{BDMS})_{2} \mathrm{Te}$ & & & DT & 41 \\
\hline $\mathrm{CdSe} / \mathrm{CdS}$ & $\mathrm{Cd}\left(\mathrm{S}_{2} \mathrm{CNMeHex}\right)_{2}$ & TOPO/TOP & & DT & 41 \\
\hline $\mathrm{CdS} / \mathrm{ZnS}$ & $\mathrm{ZnEt}_{2},(\mathrm{TMS})_{2} \mathrm{~S}$ & TOPO & TOPO & DT & 11 \\
\hline \multirow[t]{2}{*}{$\mathrm{CdSe} / \mathrm{ZnS}$} & $\mathrm{ZnEt}_{2},(\mathrm{TMS})_{2} \mathrm{~S}$ & TOPO & TOPO & DT & 11 \\
\hline & $\mathrm{Zn}\left(\mathrm{S}_{2} \mathrm{CNMeHex}\right)_{2}$ & TOPO/TOP & & DT & 41 \\
\hline $\mathrm{CdSe} / \mathrm{ZnSe}$ & $\mathrm{Zn}\left(\mathrm{Se}_{2} \mathrm{CNMeHex}\right)_{2}$ & TOPO/TOP & & DT & 41 \\
\hline
\end{tabular}

DT, decomposição térmica 
$\mathrm{CdSe}^{49}$ e $\mathrm{CdTe}^{50}$ passivados com tióis. Estas sínteses geralmente envolvem um sal solúvel de $\mathrm{Cd}$ (II) e um composto calcogenoide, que reagem na presença de tiolatos; estes atuam de modo semelhante às moléculas de TOPO já descritas, no entanto, tratando-se de moléculas ambidentadas, os grupos tiol coordenam à superfície dos QD e os grupos terminais $\left(-\mathrm{OH},-\mathrm{COOH}\right.$ ou $-\mathrm{NH}_{2}$ ) conferem compatibilidade com a água.

\section{Síntese de nanopartículas metálicas}

Entre as NP metálicas, ouro e prata são sistemas especialmente investigados. Os métodos convencionais de síntese de NP de ouro baseiam-se na redução do correspondente sal de ouro (III), podendo obter-se coloides estáveis aquosos ou em meio orgânico.

O método mais comum foi introduzido por Turkevich, em 1951, ${ }^{15}$ e utiliza soluções aquosas em que o ião citrato é agente redutor de $\mathrm{HAuCl}_{4}$ e estabilizante coloidal por intermédio de repulsões eletroestáticas. Apesar de inicialmente este método ter estado limitado a NP de cerca de $20 \mathrm{~nm}$, posteriormente foi adaptado a sínteses de NP com tamanhos médios de 16 a $147 \mathrm{~nm}$, variando-se a razão entre o reagente de ouro e o agente estabilizador/redutor. ${ }^{51}$ A ligação do ião citrato à superfície da NP é relativamente fraca, o que é particularmente interessante quando se deseja proceder a reacções de substituição à superfície. A introdução de agentes anfifílicos baseados em tióis conjuntamente com o citrato tem sido também apresentada com sucesso. ${ }^{52}$

Brust e Schiffrin descreveram a obtenção de NP de Au com dimensões mais reduzidas (diâmetros entre 1,5 e 5,2 nm) com boa estabilidade térmica e coloidal. ${ }^{53}$ Estes investigadores desenvolveram um método que utiliza $\mathrm{NaBH}_{4}$ como agente redutor do ouro(III) $\left(\mathrm{HAuCl}_{4}\right)$ num sistema de duas fases: tolueno/água. ${ }^{53} \mathrm{O}$ ião $\mathrm{AuCl}_{4}{ }^{-}$é transferido para a fase orgânica pela ação de um tensioativo (brometo de tetraoctilamónio, TOAB) o que permite a redução de ouro iónico pelo $\mathrm{NaBH}_{4}$. A ligação do TOAB à superfície das NP formadas não é suficientemente forte para garantir estabilidade coloidal a longo prazo. A estabilidade do coloide é promovida pela adição de tióis de cadeia carbonada longa que, devido à afinidade do Au (ácido mole) pelo $\mathrm{S}$ (base mole), coordenam fortemente à superfície das NP. ${ }^{53,54}$ Posteriormente, os mesmos autores desenvolveram um método de síntese de NP de ouro estabilizadas por $p$-mercaptofenol em sistema monofásico ${ }^{54}$ Este trabalho tem sido uma referência na preparação de uma grande variedade de NP de ouro estabilizadas por tióis. ${ }^{55}$

Murphy e colaboradores ${ }^{56}$ obtiveram NP de Au com tamanhos médios entre 5-40 nm, utilizando agentes redutores distintos na preparação de sementes e na fase de crescimento em partículas maiores. As sementes de ouro foram preparadas por redução de $\mathrm{HAuCl}_{4}$ utilizando $\mathrm{NaBH}_{4}$ na presença de ião citrato e, na fase de crescimento, adicionou-se ácido ascórbico. A estabilidade das partículas durante a fase de crescimento foi promovida pela adição do agente tensioativo brometo de cetiltrimetilamónio, CTAB. O ácido ascórbico é um redutor fraco na redução de espécies de $\mathrm{Au}(\mathrm{III})$ a Au coloidal, em solução aquosa. No entanto, na presença de sementes de Au formadas previamente na presença de $\mathrm{NaBH}_{4}$, revelou-se bastante eficaz no crescimento controlado dessas sementes. ${ }^{56}$

Perrault e colaboradores ${ }^{57}$ conseguiram aumentar o tamanho médio de NP de ouro, preparadas por redução com ião citrato, até uma gama de tamanhos de 50 a $200 \mathrm{~nm}$, mantendo-se monodispersas, utilizando um agente redutor mais suave como a hidroquinona na fase de crescimento de partícula. O ião citrato adsorvido na superfície das NP garante o crescimento controlado das NP coloidais estabilizadas eletroestaticamente. Inicialmente produzem-se sementes de NP de ouro, pelo método do citrato, e o crescimento posterior é garantido pela ação catalítica da hidroquinona à superfície das sementes previamente formadas.
A redução do sal do metal também pode ser efetuada por ação de um glicol como, por exemplo, o etanodiol-1,2 (etilenoglicol). Este método foi proposto originalmente na síntese de partículas monodispersas e micrométricas para vários metais, ${ }^{58}$ tendo sido adaptado à obtenção de NP utilizando solventes tensioativos como a oleilamina ou o ácido oleíco. A oleilamina pode atuar simultaneamente como solvente e agente redutor, como demonstrado na preparação de NP de Ag. ${ }^{59}$ A Tabela 2 ilustra condições de síntese típicas na preparação de NP de $\mathrm{Au}$ e Ag por diversos métodos em solução.

Tabela 2. Condições de síntese de NP de Au e Ag pela redução dos respetivos sais

\begin{tabular}{|c|c|c|c|c|}
\hline Material & Reagentes & Estabilizantes & Solvente & Ref. \\
\hline \multirow[t]{8}{*}{$\mathrm{Au}$} & $\mathrm{HAuCl}_{4}$, citrato & Citrato & Água & 15,51 \\
\hline & $\mathrm{HAuCl}_{4}$, citrato & tiois & Água & 52 \\
\hline & $\mathrm{HAuCl}_{4}, \mathrm{NaBH}_{4}$ & TOAB, DDT & tolueno/água & 53 \\
\hline & $\mathrm{HAuCl}_{4}, \mathrm{NaBH}_{4}$ & $\begin{array}{l}p \text {-mercapto } \\
\text { fenol }\end{array}$ & Metanol & 54 \\
\hline & $\begin{array}{l}\mathrm{HAuCl}_{4}, \mathrm{NaBH}_{4} \text {, } \\
\text { ac. ascórbico }\end{array}$ & Citrato/CTAB & Água & 56 \\
\hline & $\begin{array}{c}\mathrm{HAuCl}_{4} \text {, citrato, } \\
\text { hidroquinona }\end{array}$ & Citrato/CTAB & Água & 57 \\
\hline & $\begin{array}{l}\mathrm{HAuCl}_{4}, \mathrm{Na}_{3} \mathrm{Cit} \text {, } \\
\text { acrilato de sódio }\end{array}$ & $\begin{array}{l}\text { Acrilato de } \\
\text { sódio, } \\
\text { Ac. acrílico }\end{array}$ & Água & 60 \\
\hline & $\mathrm{HAuCl}_{4}, \mathrm{EG}$ & $\begin{array}{l}\text { OAm, OAc, } \\
\text { PVP }\end{array}$ & Água & 61 \\
\hline $\mathrm{Ag}$ & $\mathrm{Ag}\left(\mathrm{NO}_{3}\right), \mathrm{OAm}$ & OAm & Parafina & 59 \\
\hline
\end{tabular}

\section{Síntese de nanopartículas magnéticas}

As NP de óxidos de ferro, $\gamma-\mathrm{Fe}_{2} \mathrm{O}_{3}$ (maguemite) e $\mathrm{Fe}_{3} \mathrm{O}_{4}$ (magnetite), têm sido muito utilizadas em bioaplicações como, por exemplo, em imagiologia de ressonância magnética, libertação controlada de fármacos e em hipertermia. Na forma macrocristalina, a magnetite e a maguemite têm a estrutura de espinela invertida, tendo a maguemite defeitos catiónicos, e são ambas ferrimagnéticas. ${ }^{24}$

Um método comum de preparação de óxidos ferrimagnéticos consiste na coprecipitação de sais de Fe(II) e Fe(III) em meio alcalino, usando soluções aquosas de $\mathrm{NaOH}$ ou amônia: ${ }^{62}$

$$
\mathrm{Fe}^{2+}+2 \mathrm{Fe}^{3+}+8 \mathrm{OH}^{-} \stackrel{\mathrm{H}_{2} \mathrm{O}}{\longrightarrow} \mathrm{Fe}_{3} \mathrm{O}_{4}+4 \mathrm{H}_{2} \mathrm{O}
$$

Este método é vantajoso pela sua simplicidade e permite a preparação de grandes quantidades de óxidos de ferro por métodos amigos do ambiente. A maior limitação prende-se com a dificuldade no controle da distribuição de tamanhos de partículas, obtendo-se geralmente amostras polidispersas. A adição de citrato, ${ }^{63}$ hidrazina ${ }^{64}$ ou ácido oleíco ${ }^{65}$ promove a estabilidade coloidal e, no último caso, torna as NP compatíveis com solventes não polares.

A decomposição térmica de precursores de ferro em solventes tensioativos de ponto de ebulição elevado é o método mais indicado para a preparação de NP monodispersas de óxidos de ferro, com diâmetros que podem variar entre 3 e $50 \mathrm{~nm}$. Neste contexto, têm sido usados precursores como $\mathrm{Fe}(\mathrm{CO})_{5}, \mathrm{Fe}$ (acetilacetonato) ${ }_{3} \mathrm{e}$ $\mathrm{Fe}$ (cupferronato) ${ }_{3}$, entre outros. ${ }^{66-71}$

As NP de ligas metálicas de ferro e platina, assim como de cobalto e platina, têm sido obtidas a partir da redução dos sais em glicóis. Murray e colaboradores sintetizaram NP de FePt, por redução de $\mathrm{Pt}(\mathrm{acac})_{2}$, utilizando 1,2-hexadecanodiol como agente redutor, e por 
Tabela 3. Condições de síntese de NPs com propriedades magnéticas

\begin{tabular}{|c|c|c|c|c|c|}
\hline Material & Precursores & surfactantes & solventes & Método & Ref \\
\hline \multirow[t]{2}{*}{$\mathrm{CoPt}$} & $\mathrm{Co}(\mathrm{CO})_{3} \mathrm{NO}, \mathrm{Pt}(\mathrm{acac})_{2}, \mathrm{HDD}$ & OAc, OAm & $\mathrm{OE}$ & DT, R & 73 \\
\hline & $\mathrm{Co}(\mathrm{Ac})_{2}, \mathrm{Pt}(\mathrm{acac}) 2, \mathrm{PEG} 200$ & OAc, OAm & $\mathrm{Ph} 2 \mathrm{O}$ & $\mathrm{R}$ & 74 \\
\hline \multirow[t]{3}{*}{$\mathrm{FePt}$} & $\mathrm{Fe}(\mathrm{CO})_{5}, \mathrm{Pt}(\mathrm{acac}) 2, \mathrm{HDD}$ & OLEA, OAm, HDD & $\mathrm{OE}$ & DT, R & 75 \\
\hline & $\mathrm{Fe}(\mathrm{CO})_{5}, \mathrm{Pt}(\mathrm{acac})_{2}$ & OLEA, OAm & éter dibenzílico & DT & 72 \\
\hline & $\mathrm{Fe}(\mathrm{acac})_{2}, \mathrm{Pt}(\mathrm{acac})_{2}, \mathrm{HDD}$ & OLEA, OAm, HDD & $\mathrm{OE}$ & $\mathrm{R}$ & 76 \\
\hline $\mathrm{CoPt}_{3}$ & $\begin{array}{c}\mathrm{Co}_{2}(\mathrm{CO})_{8}, \mathrm{Pt}(\mathrm{acac})_{2}, \mathrm{HDD} \\
\mathrm{Co}(\mathrm{CO})_{3} \mathrm{NO}, \mathrm{Pt}(\mathrm{acac})_{2}, \mathrm{HDD}\end{array}$ & $\begin{array}{l}\text { ACA, HDA } \\
\text { OAc, OAm }\end{array}$ & $\begin{array}{l}\text { PE, ODCB } \\
\text { OE }\end{array}$ & $\begin{array}{l}\text { DT, R } \\
\text { DT, R }\end{array}$ & $\begin{array}{l}77 \\
73\end{array}$ \\
\hline $\mathrm{FeCoPt}$ & $\mathrm{Fe}(\mathrm{CO})_{5}, \mathrm{Pt}(\mathrm{acac})_{2}, \mathrm{Co}(\mathrm{acac})_{2}, \mathrm{HDD}$ & OLEA, OAm, HDD & $\mathrm{OE}$ & DT, R & 78 \\
\hline \multirow[t]{3}{*}{$\mathrm{MnO}$} & acetato de manganês & OAc & & DT & 79 \\
\hline & $\operatorname{Mn}(\mathrm{acac})_{2}$ & $\mathrm{OAm}, \mathrm{H}_{2} \mathrm{O}$ & & DT & 80 \\
\hline & $\mathrm{Mn}_{2}(\mathrm{CO})_{10}$ & OAm, TOP & & DT & 81 \\
\hline \multirow[t]{7}{*}{$\gamma-\mathrm{Fe}_{2} \mathrm{O}_{3}$} & $\mathrm{Fe}(\mathrm{Cup})_{3}$ & TOA, OA & & DT & 66 \\
\hline & $\mathrm{Fe}(\mathrm{CO})_{5}$, TMANO & $\mathrm{OAc}$ & $\mathrm{OE}$ & DT & 67 \\
\hline & $\mathrm{Fe}(\mathrm{CO})_{5}$, ar & $\mathrm{OAc}$ & $\mathrm{OE}$ & DT & 69 \\
\hline & $\mathrm{Fe}(\mathrm{acac})_{3,}, \mathrm{HDD}$ & OAc, OAm, HDD & $\mathrm{PE}$ & $\mathrm{R}$ & 70 \\
\hline & $\mathrm{FeCl}_{3} \cdot 6 \mathrm{H}_{2} \mathrm{O}, \mathrm{FeCl}_{3} \cdot 4 \mathrm{H}_{2} \mathrm{O}$ & $\mathrm{OAc}$ & ODE & DT & 71 \\
\hline & $\mathrm{FeO}(\mathrm{OH})$ & $\mathrm{OAc}$ & ODE & DT & 32 \\
\hline & $\mathrm{FeCl}_{3} \cdot 6 \mathrm{H}_{2} \mathrm{O}, \mathrm{Na}(\mathrm{OAc})$ & $\mathrm{OAc}$ & ODE & DT & 30 \\
\hline \multirow[t]{8}{*}{$\mathrm{Fe}_{3} \mathrm{O}_{4}$} & $\mathrm{Fe}(\mathrm{II}), \mathrm{Fe}(\mathrm{III}), \mathrm{NaOH} / \mathrm{NH}_{3}$ & & Aq. & $\mathrm{H}$ & 62 \\
\hline & $\mathrm{Fe}(\mathrm{II}), \mathrm{Fe}(\mathrm{III}), \mathrm{NaOH} / \mathrm{NH}_{3}$ & Citrato & Aq. & $\mathrm{H}$ & 63 \\
\hline & $\mathrm{Fe}(\mathrm{II}), \mathrm{Fe}(\mathrm{III}), \mathrm{NaOH} / \mathrm{NH}_{3}$ & Hidrazina & Aq. & $\mathrm{H}$ & 64 \\
\hline & $\mathrm{Fe}(\mathrm{II}), \mathrm{Fe}(\mathrm{III}), \mathrm{NaOH} / \mathrm{NH}_{3}$ & $\mathrm{OAc}$ & Aq. & $\mathrm{H}$ & 65 \\
\hline & $\mathrm{Fe}(\mathrm{CO})_{5}, \mathrm{OAc}, \mathrm{TMNO}$ & $\mathrm{OAc}$ & $\mathrm{OE}$ & DT, Ox. & 82 \\
\hline & Oleato de Ferro & $\mathrm{OAc}$ & ODE & DT & 71 \\
\hline & $\mathrm{Fe}(\mathrm{acac})_{3}, \mathrm{HDD}$ & OAc, OAm & DPE & DT, R & 70 \\
\hline & $\mathrm{Fe}(\mathrm{acac})_{3,} \mathrm{OAm}$ & OAm & Éter benzílico & $\mathrm{R}$ & 83 \\
\hline
\end{tabular}

DT, Decomposição térmica; R, redução; $H$, hidrólise; Ox. Oxidação

decomposição simultânea de $\mathrm{Fe}(\mathrm{CO})_{5}$, na presença de ácido oleíco e oleilamina. Sun e colaboradores utilizaram éter benzílico como solvente e ácido oleíco e oleilamina como estabilizantes para a preparação de NP de ligas de FePt numa reação de um só passo a partir de $\left[\mathrm{Fe}(\mathrm{CO})_{5}\right]$ e $\left[\mathrm{Pt}(\mathrm{acac})_{2}\right]$. O tamanho, a composição e forma das NP foram controlados manipulando-se certos parâmetros, como a razão molar dos estabilizantes relativamente aos precursores metálicos, velocidade de aquecimento e temperatura de reação. ${ }^{72}$

Weller e colaboradores descreveram a síntese de $\mathrm{NP}$ de $\mathrm{CoPt}_{3}$ por um método semelhante, a partir da decomposição de $\left[\mathrm{Co}_{2}(\mathrm{CO})_{8}\right] \mathrm{e}$ redução simultânea de $\left[\mathrm{Pt}(\mathrm{acac})_{2}\right]$ com hexadecanodiol-1,2 utilizando diferentes estabilizantes. $\mathrm{O}$ tamanho das $\mathrm{NP}$ variou de 1,5 a 7,2 nm, por variação da temperatura de reação. ${ }^{77} \mathrm{~A}$ preparação de ligas de $\mathrm{Co}-\mathrm{Pt}$ nestas condições resultou na forma $\mathrm{CoPt}_{3}$, independentemente da razão molar dos precursores de cobalto e platina. Para obter a liga na forma $\mathrm{CoPt}$, Chen e colaboradores utilizaram $\mathrm{Co}(\mathrm{CO})_{3}(\mathrm{NO})$ como precursor de cobalto e $\mathrm{Pt}(\mathrm{acac})_{2}$ como precursor de $\mathrm{Pt}$, na presença de ácido oleico e oleilamina em éter octílico. Por este método é possível obter as duas estequiometrias, $\mathrm{CoPt}$ e $\mathrm{CoPt}_{3}$, simplesmente por variação da relação inicial dos precursores de cobalto e platina. ${ }^{73}$

\section{Modificação superficial de NP e derivados compósitos}

Os métodos de síntese em solventes tensioativos de elevado ponto de ebulição dão origem a NP hidrofóbicas, como resultado da passivação da superfície com as respectivas moléculas orgânicas. A transferência de fase das NP hidrofóbicas para meios aquosos, com a introdução de funcionalidades químicas passíveis de interagir com biomoléculas, é um passo importante, por exemplo, para a aplicação biológica de NP. As entidades biológicas são especialmente sensíveis ao meio, em particular às condições de pH e força iónica de soluções aquosas. Por outro lado, as NP deverão ser suficientemente estáveis em soluções aquosas, para que sejam compatíveis com os fluídos biológicos. Estes meios revelam-se por vezes agressivos para as NP, por exemplo, por processos de oxidação, pelo que a modificação superficial de NP desempenha outro papel não menos importante, ao originar barreiras que inibem a degradação química das NP. Por outro lado, em termos de bioaplicações, os processos de modificação superficial podem contribuir para limitar efeitos de citotoxicidade que eventualmente possam existir. ${ }^{84}$

A transferência de NP hidrofóbicas para soluções aquosas pode ser efetuada por modificação prévia da superfície com ligantes hidrofílicos, segundo duas vias distintas: a substituição dos ligantes originais por moléculas hidrofílicas ou pelo encapsulamento das NP em revestimentos heterofuncionais, a partir de interações hidrofóbicas com as moléculas estabilizantes.

Na primeira metodologia, a troca do ligante envolve a substituição das moléculas estabilizantes nativas, introduzidas durante o processo de síntese, por moléculas orgânicas ambidentadas. Estas são moléculas bifuncionais que, por um lado, se coordenam à superfície da NP como bases de Lewis (grupos tiol ou carboxílicos) e, por outro lado, possuem grupos hidrófilos (por exemplo, grupos carboxílicos ou amínicos) que apontam para o exterior da superfície da NP e permitem estabilidade coloidal em solução aquosa. A molécula do ligante utilizado para a substituição apresenta uma maior afinidade para a superfície da NP do que o ligante original ou, então, é utilizado em excesso facilitando a substituição por transferência de massa. Este método também é aplicável a NP sintetizadas em solução aquosa. Por exemplo, os coloides de Au estabilizados por iões citrato são estáveis por longos períodos de tempo, no entanto, são sensíveis a alterações 
do meio podendo formar agregados de forma irreversível na presença de sais iónicos. Os grupos citrato podem ser substituídos por ligantes que conferem estabilidade adicional, como os ácidos mercaptocarboxílicos ${ }^{85}$ e as fosfinas sulfonadas. ${ }^{86,87}$ As NP modificadas com fosfinas sulfonadas revelam uma grande estabilidade, permitindo que possam ser precipitadas por agregação induzida por adição de sal e novamente redispersas. O ligante fosfina pode ser então substituído por um ligante contendo um tiol, estratégia essa que é muito comum para a ligação de ADN funcionalizado com grupos tiol a NP de ouro. ${ }^{87}$ As NP de Au e Ag sintetizadas em meio orgânico são geralmente estabilizadas com tióis (dodecanotiol) ou com aminas de cadeia longa (oleilamina). Estes estabilizantes hidrofóbicos podem ser substituídos por ligantes tiolatos, ${ }^{88} \mathrm{o}$ que permite a transferência das NP para a fase aquosa, assim como a introdução de grupos funcionais.

$\mathrm{O}$ crescimento de camadas de sílica amorfa à superfície de NP constitui uma estratégia alternativa, no sentido de conferir propriedades hidrofílicas a coloides originalmente hidrofóbicos. As NP assim modificadas podem ser posteriormente funcionalizadas com outras moléculas ou polímeros. ${ }^{89} \mathrm{O}$ revestimento com sílica começa com uma reação de troca, em que os ligantes originais são substituídos por silanos, que para o caso de NP de Au poderá ser mercaptopropiltris(metiloxi)ssilano (MPS). A condensação dos grupos metoxissilano ocorre por uma reação de hidrólise em meio alcalino. O crescimento da capa de sílica ocorre através da hidrólise de um alcóxido de silício (ex. TEOS), de acordo com o método de Stöber. A reatividade da sílica amorfa tem sido estudada para uma grande variedade de aplicações, existindo um vasto conhecimento na sua funcionalização química. ${ }^{90}$

A literatura descreve inúmeros trabalhos sobre a modificação superficial de NP hidrofóbicas com camadas finas de $\mathrm{SiO}_{2}$ para uma grande variedade de materiais como, por exemplo, $\mathrm{Fe}_{3} \mathrm{O}_{4}, \mathrm{Au}, \mathrm{Ag}$ e CdSe/ZnS. ${ }^{91}$ Nestes casos, o revestimento é realizado por reação de trimetoxissilanos (no lugar do TEOS) com a superfície das NP hidrofóbicas, em fase orgânica (tolueno), dando origem a um conjugado NP-silano. Os silanos são escolhidos para que a condensação de $\mathrm{SiO}_{2}$ seja iniciada à superfície. Os trimetoxissilanos podem reagir diretamente com a superfície de NP de óxidos metálicos (tal como o $\mathrm{Fe}_{3} \mathrm{O}_{4}$ ), em meio básico, devido à presença de grupos hidroxilo superficiais. No caso de NP de Au, Ag e CdSe/ZnS a funcionalização da superfície ocorre de forma semelhante ao método anterior, isto é, utilizando inicialmente o mercaptopropiltrimetoxissilano, MPS, que se coordena à superfície através do grupo tiol. A adição de um segundo silano termina a camada de sílica e confere assim funcionalidade às NP.

Outro método de revestimento de NP com sílica envolve a formação de microemulsões água em óleo e foi reportado para uma grande variedade de NP hidrofóbicas - CdSe, CdSe/ZnS, PbSe, $\gamma-\mathrm{Fe}_{2} \mathrm{O}_{3}$, bem como para misturas de NP na preparação de nanocompósitos multifuncionais. ${ }^{92}$ A formação de uma microemulsão resulta da utilização de um tensioativo não iónico em fase orgânica, com adição de uma base, como a amónia. As NP dispersas inicialmente na fase orgânica migram para o interior aquoso da microemulsão. A hidrólise e condensação do precursor de sílica (TEOS) ocorrem à superfície da NP hidrofóbica, devido à afinidade do TEOS hidrolisado com a superfície da NP, que vai substituir o ligante nativo. O mecanismo de incorporação da NP hidrofóbica nas esferas de sílica, pela troca do ligante nativo pelo TEOS hidrolisado, foi descrito recentemente. ${ }^{93}$

Recentemente foi reportado um método simples que permite o revestimento de NP individualizadas, com sílica mesoporosa, com um bom controle tanto da espessura da capa de sílica como da distribuição final de tamanhos. O processo de revestimento é iniciado por uma etapa de transferência de fase, em que as NP inicialmente preparadas em meio orgânico são transferidas para a fase aquosa, recorrendo a soluções concentradas em CTAB. A estabilidade das NP em solução aquosa é garantida pela formação de uma bicamada constituída pelas moléculas surfactantes, introduzidas durante a síntese das NP (geralmente ácido oleíco ou TOPO) e as moléculas de CTAB. A adição de TEOS à suspensão aquosa das NP, em meio alcalino, leva à formação da capa de sílica. Devido ao caráter ligeiramente hidrofóbico da molécula de TEOS, a reação de hidrólise ocorre preferencialmente na interface entre as moléculas de surfactante, isto é, à superfície das NP. ${ }^{94}$

A modificação superficial de NP utilizando polímeros, formando partículas compósitas, conduz a uma nova classe de materiais com diversas aplicações. ${ }^{95}$ Adicionalmente às propriedades singulares das NP, há interesse em explorar efeitos sinergísticos que surgem da combinação das NP inorgânicas com a matriz polimérica. Por exemplo, a estabilidade coloidal de QD revestidos com moléculas orgânicas, garantida em meios orgânicos pelos estabilizantes nativos na superfície, pode ser explorada para preparar nanocompósitos poliméricos por um processo de polimerização in situ por miniemulsão, na presença dos QD. Esta técnica foi estudada para uma grande variedade de materiais inorgânicos, tais como $\mathrm{TiO}_{2},{ }^{96} \mathrm{SiO}_{2},{ }^{97}$ e pós de carbono negro, ${ }^{98} \mathrm{em}$ que as partículas são dispersas na fase orgânica, constituída pelo monómero, sem tratamento prévio da superfície. A encapsulação de diversas NP inorgânicas com propriedades hidrofóbicas por métodos de polimerização por miniemulsão in situ foi demonstrada para poli(estireno) (PS), ${ }^{99,100}$ poli(acrilato de butilo) (PBA) ${ }^{99,101}$ e copolímeros de poli(estireno) e poli(metacrilato de metilo) (PS/PMMA). ${ }^{102}$ No caso dos nanocompósitos de QD em matrizes poliméricas, o comportamento ótico dos nanocompósitos depende de um variado número de parâmetros, que inclui principalmente o tipo de polímero utilizado. Por exemplo, o estudo do comportamento ótico de QD de CdSe em PBA demonstrou que estes nanocompósitos apresentam forte luminescência quando comparados com os nanocompósitos análogos com PS. ${ }^{103}$

Deve-se salientar que nestas estratégias não ocorre a formação de ligações covalentes fortes entre as moléculas passivantes e as moléculas do polímero. De fato, as condições experimentais devem ser otimizadas, de forma a limitar a migração das NP para a superfície das esferas poliméricas. A funcionalização de NP com grupos polimerizáveis ancorados à superfície poderá permitir a copolimerização com monómeros vinílicos, favorecendo a distribuição homogénea das NP no interior dos polímeros de látex. A copolimerização em miniemulsão de QD funcionalizados com grupos vinilo com estireno também foi investigada. A introdução do grupo vinilo na superfície dos QD ocorre por troca do ligante nativo por 4-mercaptovinilbenzeno e os QD assim funcionalizados foram dispersos em gotas de estireno. Apesar de neste caso ocorrer ligação entre os QD e o polímero, ainda se observa migração dos QD para a superfície, o que é acompanhado por uma deterioração da fotoluminescência do material final. ${ }^{100} \mathrm{De}$ forma a tentar minimizar o efeito de deterioração das propriedades de emissão por fotoluminescência, foi descrito um método em duas etapas que contempla, num primeiro passo, a formação de uma camada de sílica na superfície dos QD, seguido de um processo de copolimerização à superficie. Esta reação de copolimerização ocorre através da funcionalização da superfície de sílica com 3-metacriloxi-propil-trimetoxi-silano (MPTMS) que possui grupos polimerizáveis. Os resultados obtidos são satisfatórios, ocorrendo apenas uma pequena diminuição da intensidade da luminescência. ${ }^{104}$

A ampla distribuição de tamanhos dos materiais compósitos e as dificuldades em obter distribuições homogéneas de NP no interior das partículas poliméricas, por processos acima descritos, têm suscitado interesse no desenvolvimento de métodos de polimerização iniciada na superfície da NP funcionalizada. Apesar da sua implementação a larga escala ser ainda um desafio, esta metodologia foi demonstrada 


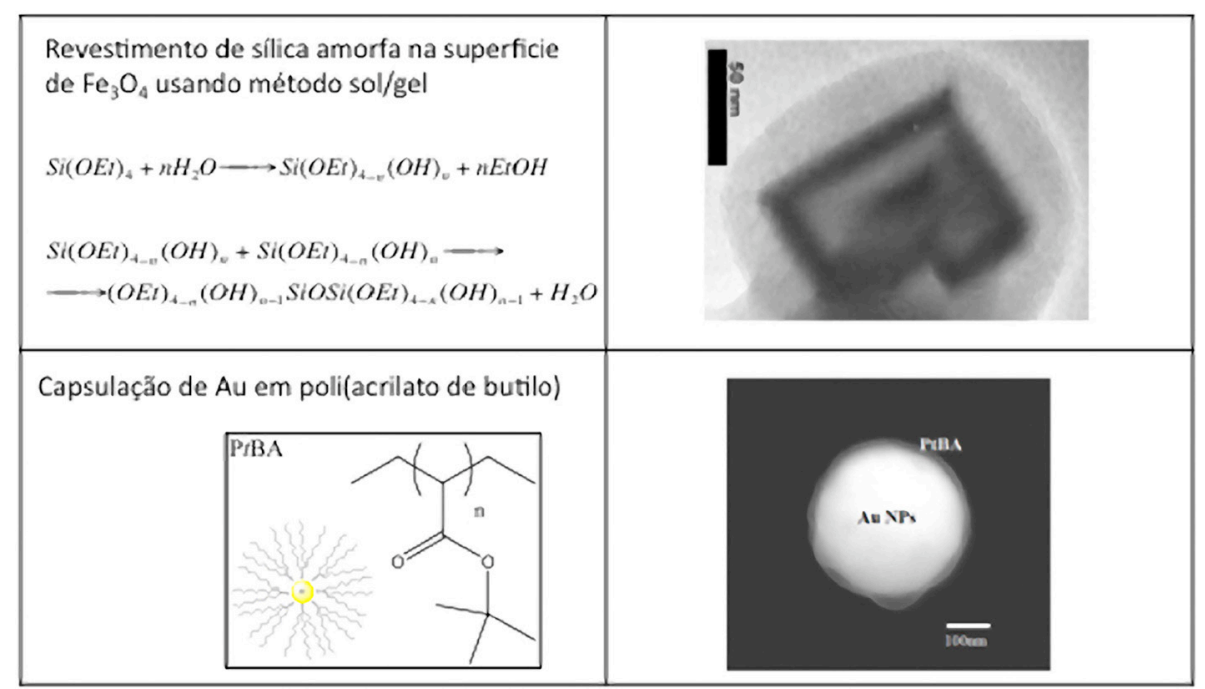

Figura 11. Modificação superficial de NP utilizando o método sol/gel e polimerização in situ. ${ }^{111}$ Imagens de microscopia das NP modificadas: $\mathrm{Fe}_{3} \mathrm{O}_{4} / \mathrm{SiO}_{2}$ e $\mathrm{AuPtBA}$

com sucesso para o crescimento controlado de cadeias poliméricas a partir da superfície de várias NP inorgânicas, tais como ouro, ${ }^{105}$ $\mathrm{SiO}_{2},{ }^{106}$ e magnetite. ${ }^{107}$ Mais recentemente, estes métodos de polimerização iniciada à superfície foram aplicados a QD. Assim, tem havido um desenvolvimento intenso destas técnicas aplicadas especificamente à superfície de $\mathrm{QD}$, tais como a polimerização controlada por reações reversíveis de adição-fragmentação de agentes de transferência de cadeia (RAFT), ${ }^{108}$ polimerização mediada com nitróxidos (NMP) ${ }^{109}$ e polimerização radicalar por transferência de átomo (ATRP). ${ }^{110}$ A Figura 11 ilustra dois métodos de modificação superficial de NP discutidos nesta seção.

\section{CONCLUSÕES}

Este artigo pretendeu sumariar o estado da arte da química preparativa de NP inorgânicas de natureza diversa, apresentando-se fundamentalmente casos ilustrativos da síntese e modificação superficial de NP como integrando uma área do conhecimento recente, frequentemente designada por Nanoquímica, mas já estabelecida ao nível do conhecimento químico atual. Se por um lado existem aspetos ao nível da química de NP que se encontram sedimentados, como o ajuste de determinadas propriedades dependentes do tamanho de partícula e efeitos de superfície, muito há ainda por explorar neste campo. Pode-se afirmar que tendencialmente as NP vão migrando da bancada do químico para investigação de cariz mais tecnológico, visando aplicações diversas. Alguns sistemas indicados ao longo deste trabalho referem-se a materiais para os quais existem estratégias preparativas e de modificação superficial bem estabelecidas na literatura. Efetivamente, alguns desses nanomateriais são comercializados ou integram produtos disponíveis no mercado. Tratou-se, neste caso, de uma opção que visa sublinhar a possibilidade de se poder utilizar NP inorgânicas como reagentes de partida na síntese de novos materiais. Apesar do progresso verificado na síntese de NP inorgânicas, a obtenção de nanomateriais de qualidade elevada de sistemas mais complexos como, por exemplo, semicondutores dopados ou fases ternárias, permanece um desafio atual. Para além disso, a literatura recente contempla um vasto leque de estudos em diferentes especialidades da Química, nos quais os efeitos de tamanho e forma são determinantes, a par de outros parâmetros normalmente investigados. Neste sentido afigura-se relevante a compreensão de processos fundamentais que ocorrem não apenas em NP mas também na sua interação com sistemas vizinhos, nomeadamente ao nível biológico e ambiental. A elaboração de novos nanomateriais, como estruturas híbridas supramoleculares e compósitas, é igualmente um tópico atual em que NP funcionalizadas são entendidas pelo químico como uma nova classe de reagentes. A este respeito, refira-se a recente revisitação da Tabela Periódica em que os agregados (clusters) surgem como novas unidades estruturais químicas na elaboração de novos materiais. ${ }^{112}$

\section{AGRADECIMENTOS}

Trabalho realizado no seguimento de estudos de doutoramento de M. Martins (bolsa da FCT: SFRH/BD/29475/2006) e no âmbito do projeto financiado pela FCT: PTDC/QUI/67712/2006.

\section{REFERÊNCIAS}

1. http://www.zyvex.com/nanotech/feynman.html, acessada em Maio 2012.

2. http://www-03.ibm.com/ibm/history/exhibits/vintage/vintage_ technology.html, acessada em Maio 2012.

3. Eigler, D. M.; Schweizer, E. K.; Nature 1990, 344, 524.

4. http://toxipedia.org/download/attachments/5998572/Undestanding_ nanoRisk_Assessment.pdf, acessada em Maio 2012.

5. Klabunde, K. J. Em Nanoscale Materials in Chemistry; Klabunde, K. J., ed.; Wiley-Interscience: New York, 2001.

6. Rossetti, R.; Ellison, J. L.; Gibson, J. M.;Brus, L. E.; J. Chem. Phys. 1984, 80, 4464.

7. Ekimov, A. I.; Efros, A. L.;Onushchenko, A. A.; Solid State Commun. 1985, 56, 921; Ekimov, A. I.;Onushchenko, A. A.; Sov. Phys. Semicond. 1982, 16, 775 .

8. Parak, W. J.; Manna, L.; Simmel, F. C.; Gerion, D.; Alivisatos, P. Em Nanoparticles: From Theory to Application; Schmid, G., ed.; WileyVCH Verlag GmbH \& Co.: Weinheim, 2004

9. Han, M. Y.; Gao, X. H.; Su, J. Z.; Nie, S.; Nat. Biotechnol. 2001, 19, 631; Chan, W. C. W.; Maxwell, D. J.; Gao, X. H.; Bailey, R. E.; Han, M. Y.; Nie, S. M.; Curr. Opin. Biotech. 2002, 13, 40; Zrazhevskiy, P.; Gao, X. H.; Nano Today 2009, 4, 414.

10. Dabbousi, B. O.; Rodriguez-Viejo, J.; Mikulec, F. V.; Heine, J. R.; Mattoussi, H.; Ober, R.; Jensen, K. F.; Bawendi, M. G.; J. Phys. Chem. B 1997, 101, 9463.

11. Smith, A. M.; Nie, S. M.; Acc. Chem. Res. 2010, 43, 190.

12. Ghosh, S. K.; Pal, T., Chem. Rev. 2007, 107, 4797.

13. Faraday, M.; Philos. Trans. Royal Soc. London 1857, 147, 145. 
14. Edwards, P. P.; Thomas, J. M.; Angew. Chem., Int. Ed. 2007, 46, 7.

15. Turkevich, J.; Stevenson, P. C.; Hillier, J.; Faraday Discuss. 1951, 55.

16. Turkevich, J.; Gold Bull. 1985, 18, 86; Turkevich, J.; Gold Bull. 1985, 18,125 .

17. Mie, G.; Ann. Phys. (Leipzig) 1908, 25, 377.

18. Liz-Marzán, L. M.; Mater. Today 2004, 26.

19. Liz-Marzán, L. M.; Langmuir 2006, 22, 32.

20. Jiang, G.; Baba, A.; Ikarashi, H.; Xu, R.; Locklin, J.; Kashif, K. R.; Shinbo, K.; Kato, K.; Kaneko, F.; Advincula, R.; J. Phys. Chem. C 2007, 111, 18687; Kelly, K. L.; Coronado, E.; Zhao, L. L.;Schatz, G. C.; J. Phys. Chem. B 2003, 107, 668; Liz-Marzán, L. M.; Giersig, M.; Mulvaney, P.; Langmuir 1996, 12, 4329.

21. Underwood, S.; Mulvaney, P.; Langmuir 1994, 10, 3427.

22. Jain, P. K.; Qian, W.; El-sayed, M. A.; J. Phys. Chem. B 2006, 110, 136; Wei, Q.-H.; Su, K.-H.; Durant, S.; Zhang, X.; Nano Lett. 2004, 4, 1067.

23. Ung, T.; Liz-Marzán, L. M.; Mulvaney, P.; Colloids Surf., A 2002, 202, 119.

24. Jeong, U.; Teng, X. W.; Wang, Y.; Yang, H.; Xia, Y. N.; Adv. Mater. 2007, $19,33$.

25. Vossmeyer, T.; Katsikas, L.; Giersig, M.; Popovic, I. G.; Diesner, K.; Chemseddine, A.; Eychmuller, A.; Weller, H.; J. Phys. Chem. 1994, 98, 7665.

26. Spanhel, L.; Anderson, M. A.; J. Am. Chem. Soc. 1991, 113, 2826.

27. Park, J.; Joo, J.; Kwon, S. G.; Jang, Y.; Hyeon, T.; Angew. Chem., Int. Ed. 2007, 46, 4630.

28. Talapin, D. V.; Rogach, A. L.; Kornowski, A.; Haase, M.; Weller, H.; Nano Lett. 2001, 1, 207

29. Hambrock, J.; Becker, R.; Birkner, A.; Weiss, J.; Fischer, R. A.; Chem. Commun. 2002, 68; Jana, N. R.; Peng, X. G.; J. Am. Chem. Soc. 2003, 125,14280 .

30. Park, J.; An, K. J.; Hwang, Y. S.; Park, J. G.; Noh, H. J.; Kim, J. Y.; Park, J. H.; Hwang, N. M.; Hyeon, T.; Nat. Mater. 2004, 3, 891.

31. Seo, W. S.; Jo, H. H.; Lee, K.; Park, J. T.; Adv. Mater. 2003, 15, 795.

32. Yu, W. W.; Falkner, J. C.; Yavuz, C. T.; Colvin, V. L.; Chem. Commun. 2004, 2306.

33. Joo, J.; Na, H. B.; Yu, T.; Yu, J. H.; Kim, Y. W.; Wu, F. X.; Zhang, J. Z.; Hyeon, T.; J. Am. Chem. Soc. 2003, 125, 11100.

34. Murray, C. B.; Norris, D. J.; Bawendi, M. G.; J. Am. Chem. Soc. 1993, 115,8706

35. Kwon, S. G.; Piao, Y.; Park, J.; Angappane, S.; Jo, Y.; Hwang, N. M.; Park, J. G.; Hyeon, T.; J. Am. Chem. Soc. 2007, 129, 12571.

36. Trindade, T.; O'Brien, P.; Pickett, N. L.; Chem. Mater. 2001, 13, 3843; Murray, C. B.; Kagan, C. R.; Bawendi, M. G.; Ann. Rev. Mater. Res. 2000, 30, 545

37. Cozzoli, P. D.; Manna, L.; Curri, M. L.; Kudera, S.; Giannini, C.; Striccoli, M.; Agostiano, A.; Chem. Mater. 2005, 17, 1296.

38. Trindade, T.; OBrien, P.; Adv. Mater. 1996, 8, 161.

39. Trindade, T.; OBrien, P.; Zhang, X. M.; Chem. Mater. 1997, 9, 523.

40. Nair, P. S.; Radhakrishnan, T.; Revaprasadu, N.; Kolawole, G.; O’Brien, P.; J. Mater. Chem. 2002, 12, 2722.

41. Malik, M. A.; O’Brien, P.; Revaprasadu, N. Chem. Mater. 2002, 14, 2004.

42. Murcia, M. J.; Shaw, D. L.; Woodruff, H.; Naumann, C. A.; Young, B. A.; Long, E. C.; Chem. Mater. 2006, 18, 2219.

43. Yu, J. H.; Joo, J.; Park, H. M.; Baik, S. I.; Kim, Y. W.; Kim, S. C.; Hyeon, T., J. Am. Chem. Soc. 2005, 127, 5662.

44. Yu, W. W.; Peng, X. G.; Angew. Chem., Int. Ed. 2002, 41, 2368.

45. Pradhan, N.; Katz, B.; Efrima, S.; J. Phys. Chem. B 2003, 107, 13843; Efrima, S.; Pradhan, N.; C. R. Chim. 2003, 6, 1035; Pradhan, N.; Efrima, S.; J. Am. Chem. Soc. 2003, 125, 2050.

46. Peng, Z. A.; Peng, X. G.; J. Am. Chem. Soc. 2001, 123, 183.

47. Peng, X. G.; Chem. Eur. J. 2002, 8, 335.

48. Rogach, A.; Kershaw, S.; Burt, M.; Harrison, M.; Kornowski, A.; Eychmuller, A.; Weller, H.; Adv. Mater. 1999, 11, 552.
49. Rogach, A. L.; Kornowski, A.; Gao, M. Y.; Eychmuller, A.; Weller, H.; J. Phys. Chem. B 1999, 103, 3065.

50. Gao, M. Y.; Kirstein, S.; Mohwald, H.; Rogach, A. L.; Kornowski, A.; Eychmuller, A.; Weller, H.; J. Phys. Chem. B 1998, 102, 8360; Rogach, A. L.; Katsikas, L.; Kornowski, A.; Su, D.; Eychmuller, A.; Weller, H.; Phys. Chem. Chem. Phys. 1997, 101, 1668; Rogach, A. L.; Katsikas, L.; Kornowski, A.; Su, D. S.; Eychmuller, A.; Weller, H.; Phys. Chem. Chem. Phys. 1996, 100, 1772.

51. Frens, G.; Nature-Phys. Sci. 1973, 241, 20

52. Yonezawa, T.; Kunitake, T.; Colloids Surf., A 1999, 149, 193.

53. Brust, M.; Walker, M.; Bethell, D.; Schiffrin, D. J.; Whyman, R.; J. Chem. Soc. Chem. Commun. 1994, 801.

54. Brust, M.; Fink, J.; Bethell, D.; Schiffrin, D. J.; Kiely, C.; J. Chem. Soc. Chem. Commun. 1995, 1655.

55. Chen, S. W.; Langmuir 1999, 15, 7551; Chen, S. W.; Murray, R. W.; Langmuir 1999, 15, 682.

56. Jana, N. R.; Gearheart, L.; Murphy, C. J.; Langmuir 2001, 17, 6782.

57. Perrault, S. D.; Chan, W. C. W.; J. Am. Chem. Soc. 2009, 131, 17042.

58. Fievet, F.; Lagier, J. P.; Blin, B.; Beaudoin, B.; Figlarz, M.; Solid State Ionics 1989, 32-3, 198; Fievet, F.; Lagier, J. P.; Figlarz, M.; MRS Bulletin 1989, 14, 12

59. Chen, M.; Feng, Y. G.; Wang, X.; Li, T. C.; Zhang, J. Y.; Qian, D. J.; Langmuir 2007, 23, 5296.

60. Njoki, P. N.; Lim, I.-I. S.; Mott, D.; Park, H.-Y.; Khan, B.; Mishra, S.; Sujakumar, R.; Luo, J.; Zhong, C.-J.; J. Phys. Chem. C 2007, 111, 14664.

61. de la Presa, P.; Multigner, M.; de la Venta, J.; Garcia, M. A.; RuizGonzalez, M. L.; J. Appl. Phys. 2006, 100, 123915.

62. Massart, R.; C. R. Hebdo. 1980, 291, 1.

63. Bee, A.; Massart, R.; Neveu, S.; J. Magn. Magn. Mater. 1995, 149, 6.

64. Hong, R. Y.; Li, J. H.; Li, H. Z.; Ding, J.; Zheng, Y.; Wei, D. G.; J. Magn. Magn. Mater. 2008, 320, 1605.

65. Shen, L. F.; Laibinis, P. E.; Hatton, T. A.; Langmuir 1999, 15, 447.

66. Rockenberger, J.; Scher, E. C.; Alivisatos, A. P.; J. Am. Chem. Soc. 1999, 121,11595 .

67. Hyeon, T.; Lee, S. S.; Park, J.; Chung, Y.; Bin Na, H.; J. Am. Chem. Soc. 2001, 123, 12798

68. Teng, X. W.; Yang, H.; J. Mater. Chem. 2004, 14, 774; Cheon, J. W.; Kang, N. J.; Lee, S. M.; Lee, J. H.; Yoon, J. H.; Oh, S. J.; J. Am. Chem. Soc. 2004, 126, 1950; Casula, M. F.; Jun, Y. W.; Zaziski, D. J.; Chan, E. M.; Corrias, A.; Alivisatos, A. P.; J. Am. Chem. Soc. 2006, 128, 1675; Wang, X.; Zhuang, J.; Peng, Q.; Li, Y. D.; Nature 2005, 437, 121; Redl, F. X.; Black, C. T.; Papaefthymiou, G. C.; Sandstrom, R. L.; Yin, M.; Zeng, H.; Murray, C. B.; O’Brien, S. P.; J. Am. Chem. Soc. 2004, 126, 14583.

69. Woo, K.; Hong, J.; Choi, S.; Lee, H. W.; Ahn, J. P.; Kim, C. S.; Lee, S. W.; Chem. Mater. 2004, 16, 2814.

70. Sun, S. H.; Zeng, H.; J. Am. Chem. Soc. 2002, 124, 8204.

71. Jana, N. R.; Chen, Y. F.; Peng, X. G.; Chem. Mater. 2004, 16, 3931.

72. Chen, M.; Liu, J. P.; Sun, S. H.; J. Am. Chem. Soc. 2004, 126, 8394.

73. Chen, M.; Nikles, D. E.; J. Appl. Phys. 2002, 91, 8477.

74. Tzitzios, V.; Niarchos, D.; Margariti, G.; Fidler, J.; Petridis, D.; Nanotechnology 2005, 16, 287.

75. Sun, S. H.; Murray, C. B.; Weller, D.; Folks, L.; Moser, A.; Science 2000, 287, 1989.

76. Liu, C.; Wu, X. W.; Klemmer, T.; Shukla, N.; Yang, X. M.; Weller, D.; Roy, A. G.; Tanase, M.; Laughlin, D.; J. Phys. Chem. B 2004, 108, 6121.

77. Shevchenko, E. V.; Talapin, D. V.; Rogach, A. L.; Kornowski, A.; Haase, M.; Weller, H.; J. Am. Chem. Soc. 2002, 124, 11480; Shevchenko, E. V.; Talapin, D. V.; Schnablegger, H.; Kornowski, A.; Festin, O.; Svedlindh, P.; Haase, M.; Weller, H.; J. Am. Chem. Soc. 2003, 125, 9090.

78. Chen, M.; Nikles, D. E.; Nano Lett. 2002, 2, 211. 
79. Yin, M.; O’Brien, S.; J. Am. Chem. Soc. 2003, 125, 10180

80. Seo, W. S.; Jo, H. H.; Lee, K.; Kim, B.; Oh, S. J.; Park, J. T.; Angew. Chem., Int. Ed. 2004, 43, 1115.

81. Park, J.; Kang, E. A.; Bae, C. J.; Park, J. G.; Noh, H. J.; Kim, J. Y.; Park, J. H.; Park, J. H.; Hyeon, T.; J. Phys. Chem. B 2004, 108, 13594.

82. Park, J.; Lee, E.; Hwang, N. M.; Kang, M. S.; Kim, S. C.; Hwang, Y.; Park, J. G.; Noh, H. J.; Kini, J. Y.; Park, J. H.; Hyeon, T.; Angew. Chem., Int. Ed. 2005, 44, 2872.

83. Xu, Z. C.; Shen, C. M.; Hou, Y. L.; Gao, H. J.; Sun, S. S.; Chem. Mater. 2009, 21, 1778 .

84. Gupta, A. K.; Berry, C.; Gupta, M.; Curtis, A.; IEEE T. Nanobiosci. 2003, 2, 255; Berry, C. C.; Curtis, A. S. G.; J. Phys. D 2003, 36, R198; Nune, S. K.; Gunda, P.; Thallapally, P. K.; Lin, Y. Y.; Forrest, M. L.; Berkland, C. J.; Expert Opin. Drug. Del. 2009, 6, 1175.

85. Lin, S. Y.; Tsai, Y. T.; Chen, C. C.; Lin, C. M.; Chen, C. H.; J. Phys. Chem. B 2004, 108, 2134.

86. Schmid, G.; Lehnert, A.; Angew. Chem., Int. Ed. 1989, 28, 780; Weare, W. W.; Reed, S. M.; Warner, M. G.; Hutchison, J. E.; J. Am. Chem. Soc. 2000, 122, 12890.

87. Loweth, C. J.; Caldwell, W. B.; Peng, X. G.; Alivisatos, A. P.; Schultz, P. G.; Angew. Chem., Int. Ed. 1999, 38, 1808.

88. Ackerson, C. J.; Jadzinsky, P. D.; Kornberg, R. D.; J. Am. Chem. Soc. 2005, 127, 6550; Hiramatsu, H.; Osterloh, F. E.; Chem. Mater. 2004, 16, 2509.

89. Gerion, D.; Pinaud, F.; Williams, S. C.; Parak, W. J.; Zanchet, D.; Weiss, S.; Alivisatos, A. P.; J. Phys. Chem. B 2001, 105, 8861.

90. Iler, R. K.; The Chemistry of Silica: Solubility, Polymerization, Colloid and Surface Properties and Biochemistry of Silica, John Wiley \& Sons: New York, 1979.

91. Jana, N. R.; Earhart, C.; Ying, J. Y.; Chem. Mater. 2007, 19, 5074.

92. Selvan, S. T.; Patra, P. K.; Ang, C. Y.; Ying, J. Y.; Angew. Chem., Int. Ed. 2007, 46, 2448; Selvan, S. T.; Tan, T. T.; Ying, J. Y.; Adv. Mater. 2005, 17, 1620; Tan, T. T.; Selvan, S. T.; Zhao, L.; Gao, S. J.; Ying, J. Y.; Chem. Mater. 2007, 19, 3112; Yi, D. K.; Selvan, S. T.; Lee, S. S.; Papaefthymiou, G. C.; Kundaliya, D.; Ying, J. Y.; J. Am. Chem. Soc. 2005, 127, 4990.

93. Koole, R.; van Schooneveld, M. M.; Hilhorst, J.; Donega, C. D.; 't Hart, D. C.; van Blaaderen, A.; Vanmaekelbergh, D.; Meijerink, A.; Chem. Mater. 2008, 20, 2503.

94. Kim, J.; Kim, H. S.; Lee, N.; Kim, T.; Kim, H.; Yu, T.; Song, I. C.; Moon, W. K.;Hyeon, T.; Angew. Chem., Int. Ed. 2008, 47, 8438; Hu, X. G.; Zrazhevskiy, P.; Gao, X. H.; Ann. Biomed. Eng. 2009, 37, 1960.
95. Zarbin, A. J. G.; Quim. Nova 2007, 30, 1469; Esteves, A. C. C.; BarrosTimmons, A.; Trindade, T.; Quim. Nova 2004, 27, 798.

96. Erdem, B.; Sudol, E. D.; Dimonie, V. L.; El-Aasser, M. S.; J. Polym. Sci., Part A: Polym. Chem. 2000, 38, 4419; Erdem, B.; Sudol, E. D.; Dimonie, V. L.; El-Aasser, M. S.; J. Polym. Sci., Part A: Polym. Chem. 2000, 38, 4431; Erdem, B.; Sudol, E. D.; Dimonie, V. L.; El-Aasser, M. S.; J. Polym. Sci., Part A: Polym. Chem. 2000, 38, 4441.

97. Tiarks, F.; Landfester, K.; Antonietti, M.; Langmuir 2001, 17, 5775.

98. Tiarks, F.; Landfester, K.; Antonietti, M.; Macromol. Chem. Phys. 2001, 202, 51.

99. Esteves, A. C. C.; Barros-Timmons, A.; Monteiro, T.; Trindade, T.; J. Nanosci. Nanotechnol. 2005, 5, 766.

100. Joumaa, N.; Lansalot, M.; Theretz, A.; Elaissari, A.; Langmuir 2006, 22 , 1810

101. Martins, M. A.; Neves, M. C.; Esteves, A. C. C.; Girginova, P. I.; Guiomar, A. J.; Amaral, V. S.; Trindade, T.; Nanotechnology 2007, 18, 215609.

102. Fleischhaker, F.; Zentel, R.; Chem. Mater. 2005, 17, 1346

103. Peres, M.; Costa, L. C.; Neves, A.; Soares, M. J.; Monteiro, T.; Esteves, A. C.; Barros-Timmons, A.; Trindade, T.; Kholkin, A.; Alves, E., Nanotechnology 2005, 16, 1969.

104. Zhu, M. Q.; Chang, E.; Sun, J. T.; Drezek, R. A.; J. Mater. Chem. 2007, 17,800 .

105. Bao, Z. Y.; Bruening, M. L.; Baker, G. L.; J. Am. Chem. Soc. 2006, 128, 9056; Ohno, K.; Koh, K.; Tsujii, Y.; Fukuda, T.; Angew. Chem., Int. Ed. 2003, 42, 2751 .

106. Ohno, K.; Morinaga, T.; Koh, K.; Tsujii, Y.; Fukuda, T.; Macromolecules 2005, 38, 2137; Zhao, H. Y.; Kang, X. L.; Liu, L.; Macromolecules 2005, 38, 10619 .

107. Marutani, E.; Yamamoto, S.; Ninjbadgar, T.; Tsujii, Y.; Fukuda, T.; Takano, M.; Polymer 2004, 45, 2231.

108. Skaff, H.; Emrick, T.; Angew. Chem., Int. Ed. 2004, 43, 5383.

109. Sill, K.; Emrick, T.; Chem. Mater. 2004, 16, 1240.

110. Esteves, A. C. C.; Bombalski, L.; Trindade, T.; Matyjaszewski, K.; Barros-Timmons, A.; Small 2007, 3, 1230.

111. Girginova, P. I.; Daniel-da-Silva, A. L.; Lopes, C. B.; Figueira, P.; Otero, M.; Amaral, V. S.; Pereira, E.; Trindade, T.; J. Colloid Interface Sci. 2010, 345, 234; Martins, M. A.; Fateixa, S.; Girao, A. V.; Pereira, S. S.; Trindade, T.; Langmuir 2010, 26, 11407.

112. Castleman, A. W.; Khanna, S. N.; J. Phys. Chem. C 2009, 113, 2664. 